Research Article

\title{
Fault Diagnosis of Piezoelectric Sensor Patches for Vibration Control Based on Multifeature Fusion and Improved SVM
}

\author{
Tian-bing Ma $\mathbb{D}^{1},{ }^{1,2}$ Zhou Qing, ${ }^{1}$ Du Fei, ${ }^{1}$ and Liu Jian ${ }^{1}$ \\ ${ }^{1}$ College of Mechanical Engineering, Anhui University of Science and Technology, Anhui Huainan 232001, China \\ ${ }^{2}$ Anhui Key Laboratory of Mine Intelligent Equipment and Technology, Anhui University of Science and Technology, \\ Huainan 232001, China \\ Correspondence should be addressed to Tian-bing Ma; dfmtb@163.com
}

Received 27 May 2019; Revised 31 August 2019; Accepted 9 October 2019; Published 11 November 2019

Academic Editor: Mohammad A. Hariri-Ardebili

Copyright $\odot 2019$ Tian-bing Ma et al. This is an open access article distributed under the Creative Commons Attribution License, which permits unrestricted use, distribution, and reproduction in any medium, provided the original work is properly cited.

\begin{abstract}
The fault diagnosis of piezoelectric sensor patches is very important for the stability of the vibration control system and faulttolerant control technology. In order to improve the accuracy of fault self-diagnosis of piezoelectric sensor patches, singular value decomposition (SVD) and Hilbert marginal spectrum method are proposed to extract multiple features of each IMF component and conduct feature fusion, and a support vector machine (SVM) based on particle swarm optimization (PSO) is designed for fault identification of different eigenvalues. In the experiment, the broken and degumming piezoelectric patches are simulated. Firstly, under the excitation of the square wave signal with no noise signal, when the SVD value and the maximum amplitude of Hilbert marginal spectrum are used as the fusion eigenvalue together, the diagnostic results show that the recognition accuracy can reach $95 \%$, compared with the recognition accuracy of $70 \%$ and $80 \%$, respectively, when the two are used as eigenvalues alone; the recognition result under fusion eigenvalue is obviously better than that of the latter. Secondly, in order to highlight the effectiveness of this method, the aforementioned experiment is conducted under the excitation of the square wave signal interfered by $0.5 \mathrm{dBW}-1 \mathrm{dBW}$ noise signal. The experimental results show that the fault recognition of the fused eigenvalue under different noise intensity signals is still superior to that of the single eigenvalue.
\end{abstract}

\section{Introduction}

At present, the research on vibration fault diagnosis technology mostly focuses on the fault detection and diagnosis of vibration components themselves, such as bearings and rotors [1-3], although there are few studies on the fault diagnosis of vibration sensors. In fact, in the study of active vibration control, the failure of the vibration sensor has some influence on the results of closed-loop vibration controlling and the stability of the system. Piezoelectric patches are widely used in vibration active control systems because of their good positive and negative piezoelectric effects. However, in practical engineering applications, repeated deformation caused by the piezoelectric effect will lead to fracture damage of fragile piezoelectric patches, whereas excessively high working temperature or excessively long working time will lead to degumming damage of piezoelectric patches [4]. In order to ensure the effective operation of the active vibration control system, fault diagnosis of piezoelectric sensor patches is very important.

In the study of related fault diagnosis methods, Zhang et al. [5] proposed a bearing fault diagnosis method, and combined EMD and permutation entropy to extract the characteristics of bearing faults. Lu et al. [6] proposed a fault diagnosis method for rotating machinery based on a deep neural network, which had good robustness under various working noises and environments. For the research on sensor fault diagnosis, Gao and Ho. [7] proposed a new generalized estimation method for generalized systems with measured output noise, which applies the progressive estimator of the generalized nonlinear system state and output noise to sensor fault diagnosis. This method has a strong applicability, but it is less targeted. Yang et al. [8] used expert system technology to identify fault types, but the diagnosis results assisted operators in troubleshooting faults, and the accuracy of identification needs to be further studied. Yang 
et al. [9] proposed a method based on residual information for fault diagnosis of the automotive active suspension sensor. The residual information of this method is greatly disturbed by the surrounding environment, and its practicality needs to be further improved.

Although the fault diagnosis research of various sensors has reached a certain level, there are a few fault diagnosis research studies on the piezoelectric patch. At present, based on piezoelectric impedance technology, Sun et al. [10] studied the change of the virtual admittance value of the piezoelectric element and then detected the self-damage of the piezoelectric element. However, this method which had a heavy task needed to detect a large amount of piezoelectric element data. Because of the various fault types of the piezoelectric patch, such as degumming fault and fracture fault, it is difficult to obtain comprehensive fault information by using a single method to extract its characteristics. Therefore, EMD-SVD processing of signals and Hilbert marginal spectrum extraction are proposed, and both of them are taken as fusion features to achieve the purpose of feature extraction of piezoelectric patch fault information. At present, EMD-SVD technology has achieved some achievements in the field of rotating mechanism fault diagnosis. For example, Hou et al. [11] used approximate singular value decomposition to extract rolling bearing fault characteristics and designed experiments to verify the effectiveness of this method under the condition of strong noise and weak fault; using BEMD and Hilbert transform, Huang et al. [12] extracted amplitude-frequency characteristics of rotor faults and proved effective in such faults as oil film oscillation, oil film rotation, and rotor loosening.

Altogether, the research of fault diagnosis technology has reached a certain level worldwide. The application of the technology is generally in the fault diagnosis of rotating mechanisms, such as gears, bearings and rotors, or the vibration parts themselves $[13,14]$. The fault diagnosis of sensors is rarely studied, and the fault diagnosis of piezoelectric sensor patches is even less studied. Therefore, selfdiagnosis of piezoelectric patches fault is still a relatively new research field. In this study, piezoelectric sensor patches in active vibration control of stiffened plate is taken as the research object. According to the common fault characteristics of piezoelectric patches, the multifeature fusion method is adopted to extract fault features, and the PSOoptimized SVM algorithm is used to identify fault types. Finally, the advantages of this method are compared and analyzed, and the fault diagnosis scheme with the best identification effect is determined.

\section{Theoretical Analysis of Piezoelectric Sensor Patch Fault Diagnosis}

2.1. Theoretical Analysis of EMD-SVD and Hilbert Marginal Spectrum. Empirical mode decomposition (EMD) is part of the Hilbert-Huang transformation algorithm. It can carry out adaptive decomposition according to the characteristics of the signal itself and obtain several intrinsic mode functions (IMF) $[15,16]$. The specific process is as follows:
(1) For signal $x(t)$, select all local extremum points and use cubic spline function to interpolate the extremum points to fit the upper and lower envelops, and then take $m_{1}(t)$, the mean of the upper and lower envelops:

$$
h_{1}=x(t)-m_{1}(t)
$$

(2) If $h_{1}$ is IMF, it is the first IMF component of $x(t)$; otherwise, take it as the initial value and repeat step (1), and the result after $k$ operations is as follows:

$$
h_{1 k}(t)=h_{1(k-1)}(t)-m_{1 k}(t)
$$

Make $h_{1 k}$ meet IMF conditions.

(3) Make

$$
c_{1}(t)=h_{1 k}(t) \text {. }
$$

Subtract $c_{1}$ from $x(t)$, you get $r_{1}(t)$ as follows:

$$
r_{1}(t)=x(t)-c_{1}(t) .
$$

Take $r_{1}(t)$ as $x(t)$ and repeat the previous steps to get the following results:

$$
r_{m}(t)=r_{m-1}(t)-c_{m}(t) .
$$

The iteration is stopped until $r_{m}(t)$ becomes a monotone function that can no longer extract IMF components. At this time,

$$
x(t)=\sum_{i=1}^{m} c_{i}(t)+r_{m}(t) .
$$

At this point, the signal is decomposed into $\mathrm{m}$ IMF components $c_{m}(t)$ and residual $r_{m}(t)$. Hilbert transformation is performed for each IMF component $c_{m}(t)$ as given in the following expression:

$$
\hat{c}_{i}(t)=\frac{1}{\pi} \int_{-\infty}^{\infty} \frac{c_{i}(\tau)}{t-\tau} \mathrm{d} \tau .
$$

Construct the analytic signal as follows:

$$
z_{i}(t)=c_{i}(t)+j \hat{c}_{i}(t)=a_{i}(t) e^{j \varphi_{i}(t)} .
$$

Among them, $a_{i}(t)$ is the instantaneous amplitude function and $\varphi_{i}(t)$ is the instantaneous phase function. If the residual $r_{m}(t)$ is ignored, we can get the following:

$$
x(t)=\operatorname{Re} \sum_{i=1}^{m} a_{i}(t) e^{j \varphi_{i}(t)}=\operatorname{Re} \sum_{i=1}^{m} a_{i}(t) e^{j \int w_{i}(t) \mathrm{d} t} .
$$

Instantaneous frequency is expressed as follows:

$$
f_{i}(t)=\frac{1}{2 \pi} w_{i}(t)=\frac{1}{2 \pi} \frac{\mathrm{d} \varphi_{i}(t)}{\mathrm{d}(t)} .
$$

Therefore, the Hilbert spectrum can be obtained by expanding equation (9) as follows: 


$$
H(w, t)=\operatorname{Re} \sum_{i=1}^{m} a_{i}(t) e^{j \int w_{i}(t) \mathrm{d} t} .
$$

Hilbert marginal spectrum is the integral of Hilbert spectrum on the time axis, as shown in the following equation:

$$
H(w)=\int_{0}^{T} H(w, t) \mathrm{d} t .
$$

Singular value decomposition (SVD) is an algebraic feature extraction method [17]. Specifically, it is an orthogonal method. By multiplying the left and right sides of the original matrix by an orthogonal matrix, it is transformed into a diagonal matrix and the linearly dependent rows or columns of the matrix become linearly independent.

For any matrix $\mathbf{A}_{m \times n}$, its rank is $r$. If there are two orthonormal moment $\mathbf{U}$ and $\mathbf{W}$ matrices and diagonal matrix D to make the following formula valid, then equation (13) is the singular decomposition of $\mathbf{A}_{m \times n}$ :

$$
\mathbf{A}=\mathbf{U D W}^{\mathrm{T}} \text {, }
$$

Among them, $\mathbf{U}$ and $\mathbf{W}$ are orthogonal matrices and $\mathbf{D}$ is a diagonal matrix.

2.2. Fault Recognition Principle Based on PSO-SVM. The support vector machine (SVM) is a method based on the statistical learning theory, whereas traditional statistical learning is difficult to achieve the ideal learning effect in the case of small samples. To address this problem, V.Vapnik and his research team proposed the SVM. The basic idea of SVM is to define the optimal hyperplane and transform the algorithm of finding the optimal linear hyperplane in space into an optimization problem $[18,19]$.

The actual signal data belong to the problem of linear inseparability. According to the constraint conditions,

$$
y_{i}(\omega \cdot x+b) \geq 1-\zeta_{i}, \quad i=1,2, \ldots, n .
$$

Then, the hyperplane meets the following conditions:

$$
\begin{aligned}
& \min \left[\frac{1}{2}\|\omega\|^{2}+C \sum_{i=1}^{n} \xi_{i}\right], \\
& \text { s.t. } \begin{cases}y_{i}(\omega \cdot x+b) \geq 1-\xi_{i}, & i=1,2, \ldots, n, \\
\xi_{i}>0, & i=1,2, \ldots, n .\end{cases}
\end{aligned}
$$

Among them, $\xi_{i}$ is the penalty function of outlier and $C$ is the penalty coefficient.

In the SVM train function, there are two important parameters $c$ and $g ; c$ is the penalty parameter, and $g$ is the kernel parameter. Particle swarm optimization (PSO) is used to optimize the selection of these two parameters. The PSO algorithm seeks the global optimal solution by following the currently searched optimal value and evaluates the quality of solution by fitness.

The fault of different piezoelectric sensor patches is simulated, and the response signals under different excitation are collected in the experiment. The signal is decomposed by EMD, and the SVD value and Hilbert marginal spectrum of IMF components are obtained as the feature vectors. After normalization, those are, respectively, sent to the SVM for training and recognition. The crossvalidation recognition accuracy of the SVM is taken as the fitness, and the $c$ and $g$ parameters of the SVM are optimized by particle swarm optimization (PSO). Finally, the recognition accuracy of the SVM algorithm under optimal parameters of $c$ and $g$ is obtained, so as to explore the influence of different feature value extraction on the fault recognition effect. The experimental flow chart is shown in Figure 1.

\section{Extraction of Fault Characteristic Parameters of Piezoelectric Sensor Patches}

3.1. Experimental System Construction and Signal Acquisition. In order to verify the effectiveness of piezoelectric sensor fault diagnosis under multiple disturbances, an experimental system of piezoelectric sensor patches fault diagnosis for vibration control of the stiffened plate, as shown in Figure 2, is established. The experimental device consists of PC, vibrator, stiffener $600 \mathrm{~mm} \times 450 \mathrm{~mm} \times 1.5 \mathrm{~mm}$, piezoelectric patches $50 \mathrm{~mm} \times 50 \mathrm{~mm} \times 0.2 \mathrm{~mm}$, Dingyang technology SDG1032X signal generator, HVP-300B power amplifier, and usb6003NI data acquisition card. In order to obtain a larger response signal, the piezoelectric patches are pasted at the maximum first mode strain of the stiffened plate. At the same time, in order to ensure the reliability of the experimental data, two groups of experiments are designed and a pair of piezoelectric patches are pasted at the maximum firstorder mode of the stiffened plate in each group. The first group of experiments are given as follows: piezoelectric patch 1 is a normal piezoelectric patch and piezoelectric patch 2 is a nearly $50 \%$ cracked piezoelectric patch. The second group of experiments include the following: piezoelectric patch 3 is a normal piezoelectric patch (the same piezoelectric patch as piezoelectric patch 1) and piezoelectric patch 4 is degumming (only a little glue is applied at the center of the patch in the experiment), and the two piezoelectric patches are pasted symmetrically opposite to each other. The two kinds of fault piezoelectric patches are shown in Figure 3. In order to display the characteristics of degumming, white paper is used to pad them.

The first mode frequency is selected as the excitation frequency, and a sinusoidal fast frequency sweep signal with a band width of $50-300 \mathrm{~Hz}$ and a time of $20 \mathrm{~s}$ were used to analyze the frequency sweep of the stiffened plate. The results show that the first mode frequency is $210 \mathrm{~Hz}$. The actual perturbation signal may contain multiple frequency components and has harmonic characteristics. In order to simulate the vibration of the stiffened plate under multidisturbance, the square wave signal is selected as the excitation signal of the stiffened plate. Square wave signals can be superimposed by a variety of sinusoidal signals, and in addition to sinusoidal fundamental frequency signals, there are also harmonic signal components. Therefore, square wave signals with different duty ratios are selected to simulate multiple disturbances. In the experiment, a total of 20 


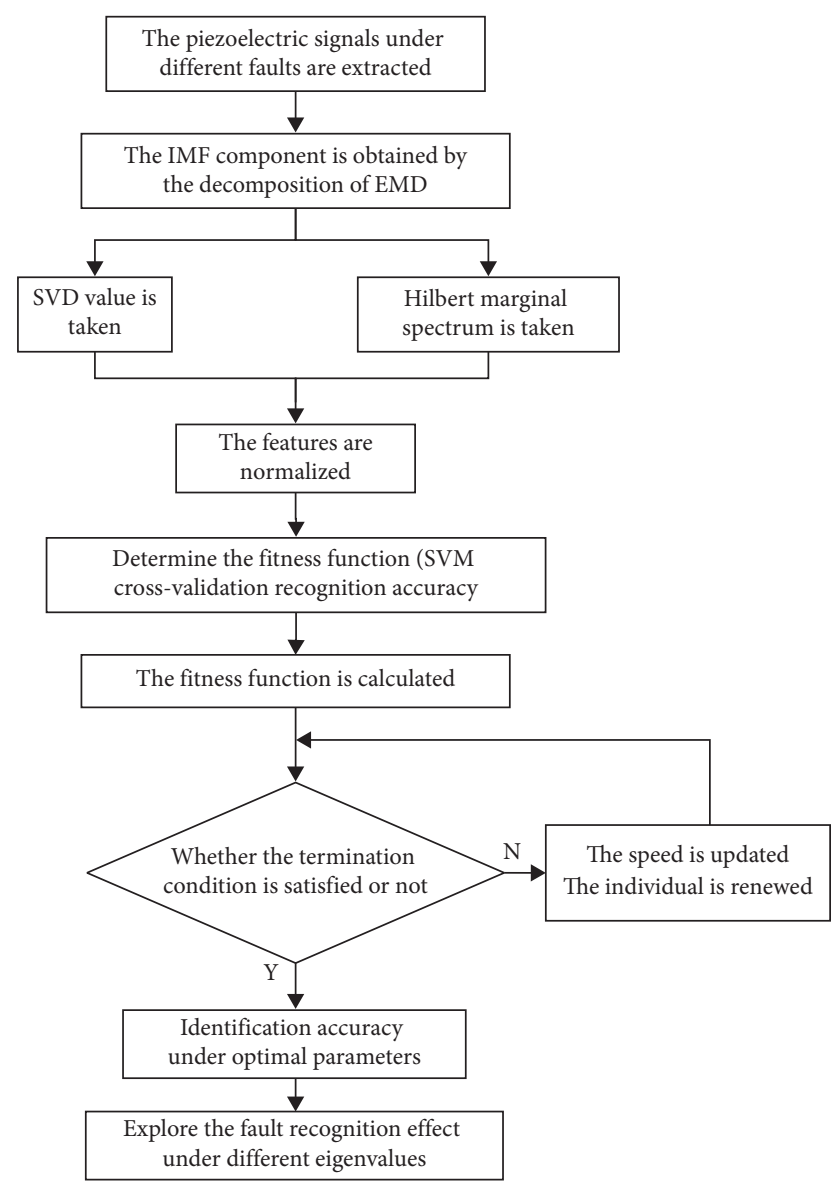

Figure 1: Experimental flow chart.

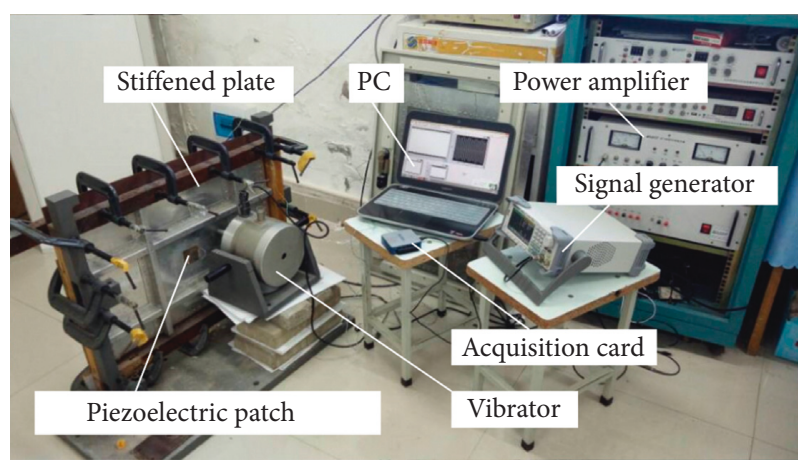

Figure 2: Physical drawing of device.

groups of square wave signals with a duty cycle of $2.5 \%$ to $50 \%$ (set at $2.5 \%$ intervals) and a frequency of $210 \mathrm{~Hz}$ are set to stimulate the stiffened plate. In order to ensure the comparability of experimental data, the response amplitude of normal patches in each group of experiments is set as $1 \mathrm{~V}$ by adjusting the power amplifier. The signals 1, 2, and 4 of the piezoelectric patches are collected as the experimental signal data of normal piezoelectric patches, broken piezoelectric patches, and degummed piezoelectric patches; a total of 60 groups of data are collected. As can be seen from the response signals of the three piezoelectric patches, although the response amplitudes of the three piezoelectric patches are different under the excitation of the same frequency, when the excitation frequency of the stiffened plate changes, the signal of piezoelectric patch will change in response. Therefore, it is difficult to distinguish the fault types of piezoelectric patches only from the response amplitude, so it is necessary to extract the fault characteristics of piezoelectric patches by means of relevant signal processing.

3.2. Fault Feature Extraction of Piezoelectric Patches Based on EMD Transform. Empirical modal decomposition (EMD) can decompose the signal into several natural modal functions adaptively according to the local characteristics of the signal itself [20-22]. The problems of choosing the optimal basis of wavelet decomposition, constant multiresolution, and energy leakage in traditional time-frequency analysis methods are fundamentally solved. At the same time, EMD can accurately obtain the global amplitude distribution of each frequency $[23,24]$.

In terms of feature extraction, SVD, as a normalized method, has good stability and is widely used in signal analysis and mechanical fault diagnosis [25-27]. The combination of EMD and SVD can overcome the randomness of choosing embedding dimension and other problems existing in the current time-frequency analysis method and can extract the energy characteristics of signals in various frequency bands well. Hilbert marginal spectrum based on Hilbert-Huang transformation, which can describe the change of the signal amplitude in the entire frequency band, is adopted to enrich the fault feature information of piezoelectric patches. The specific extraction process of piezoelectric patches fault features is as follows:

(1) three kinds of piezoelectric patches response signals under excitation of different duty cycle analog wave signals are introduced.

(2) the signals of normal piezoelectric patches, degumming piezoelectric patches, and broken piezoelectric patches are decomposed into a series of IMF components by the EMD method, respectively, and it is found that the energy is basically concentrated on the first five IMF components, as shown in Figure 4 (considering the article space problem, only IMF components of the signals of three kinds of piezoelectric patches under the excitation of the square wave with $2.5 \%$ duty cycle are listed).

(3) the SVD values of IMF components are obtained, and the SVD eigenvectors are shown in Table 1 (considering the article space problem, only the SVD values of three kinds of piezoelectric signals under $2.5 \%, 5 \%, 7.5 \%, 10 \%$, and $12.5 \%$ duty cycles are listed).

(4) Hilbert marginal spectrum is made for each component IMF, as shown in Figure 5 (considering the article space problem, only Hilbert marginal spectrum of IMF1 components of three kinds signals under the excitation of the square wave with $2.5 \%$ duty cycle is listed). The maximum point of the marginal spectrum are found, the results are shown 


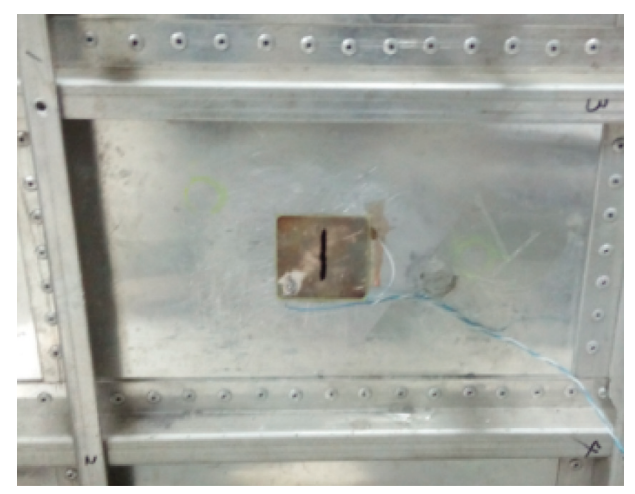

(a)

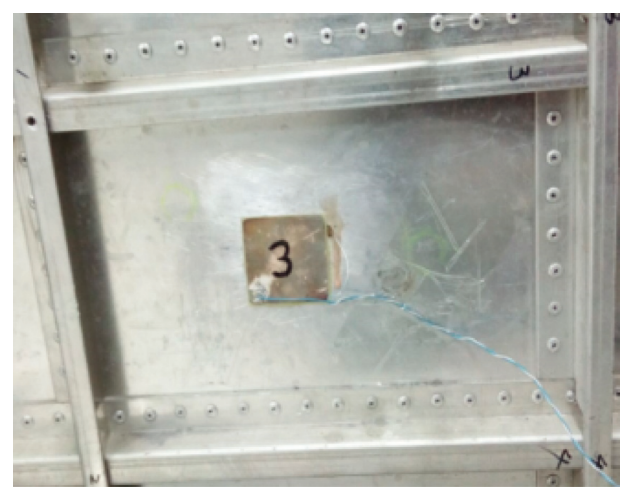

(c)

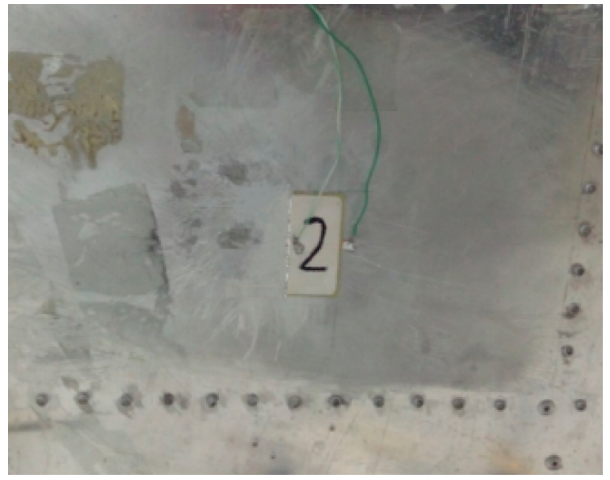

(b)

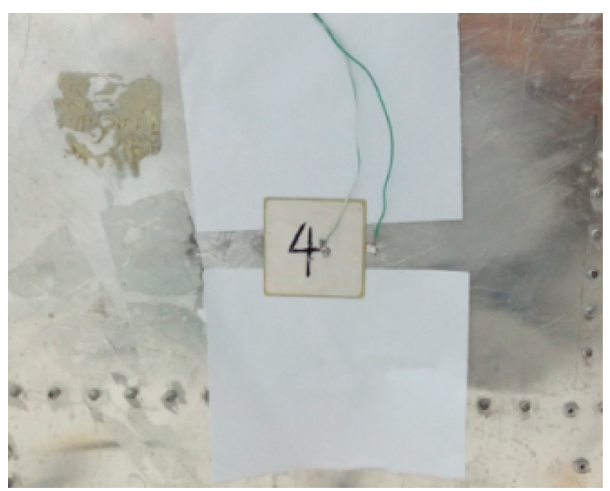

(d)

Figure 3: Piezoelectric wafer distribution: (a) normal piezoelectric patch 1 on the front; (b) broken piezoelectric patch 2 on the reverse side; (c) normal piezoelectric patch 3 on the front; (d) degummed piezoelectric patch 4 on the reverse side.

in Table 2 (only the results under the excitation of the square wave with $2.5 \%, 5 \%, 7.5 \%, 10 \%$, and $12.5 \%$ duty cycles are listed). And the result is taken as the feature vector of each piezoelectric patches signal together with the SVD value.

(5) the eigenvectors are normalized, and the new feature vector is used as the training set for the classification and recognition of subsequent algorithms.

As shown in Table 1, the signals are decomposed into IMF components from high to low EMD in frequency. The overall characteristics of the singular values of each component and Hilbert marginal spectral amplitude are not very obvious. Therefore, the support vector machine method based on particle swarm optimization parameters is used to identify the fault types of piezoelectric patches.

\section{Fault Identification of Piezoelectric Patches Based on PSO-SVM}

The support vector machine (SVM) is a new machine learning algorithm; compared with the neural network intelligent diagnosis system, which requires a large number of learning samples, the SVM has unique advantages in small sample learning and high-dimensional pattern recognition $[28,29]$. Therefore, the SVM is widely used in pattern recognition, probability density estimation, function approximation, and other fields. The classification accuracy of the SVM is closely related to the selection of function parameters [30]. Among parameters, the penalty parameter $c$ and the kernel function parameter $g$ are the main influencing parameters. At present, the selection methods for $c$ and $g$ include cross-validation, genetic algorithm, and ant colony algorithm, which are all feasible.

In this study, the particle swarm optimization algorithm is selected as the optimization algorithm to optimize the selection of $c$ and $g$ of the SVM. The recognition accuracy of the SVM algorithm under cross-validation is taken as the fitness function; $c$ and $g$ parameters are selected as particles for optimization. The number of race particles is set as 20 , and the particle dimension is used as the eigenvalue dimension. The number of iterations is set as 200, and the value range of the penalty parameter $c$ is set as $[0.01,100]$. The value range of the kernel function parameter $g$ is set as $[0.1,100]$, and the number of cross-validation is set as $K=3$. Three experiments are carried out, respectively. Each experiment included 20 groups of data of normal piezoelectric patch, rupture piezoelectric patch, and degumming piezoelectric patch, and three kinds of piezoelectric patch are defined as 1, 2, and 3. Five-dimensional SVD values, 10dimensional maximum marginal spectral points, and 15dimensional SVD and maximum marginal spectral points are selected as the eigenvalues. The PSO-optimized SVM is used to identify 60 groups of data. The fitness function is the recognition accuracy of cross-validation of all data. After 200 iterations, the optimal and average recognition accuracy of 

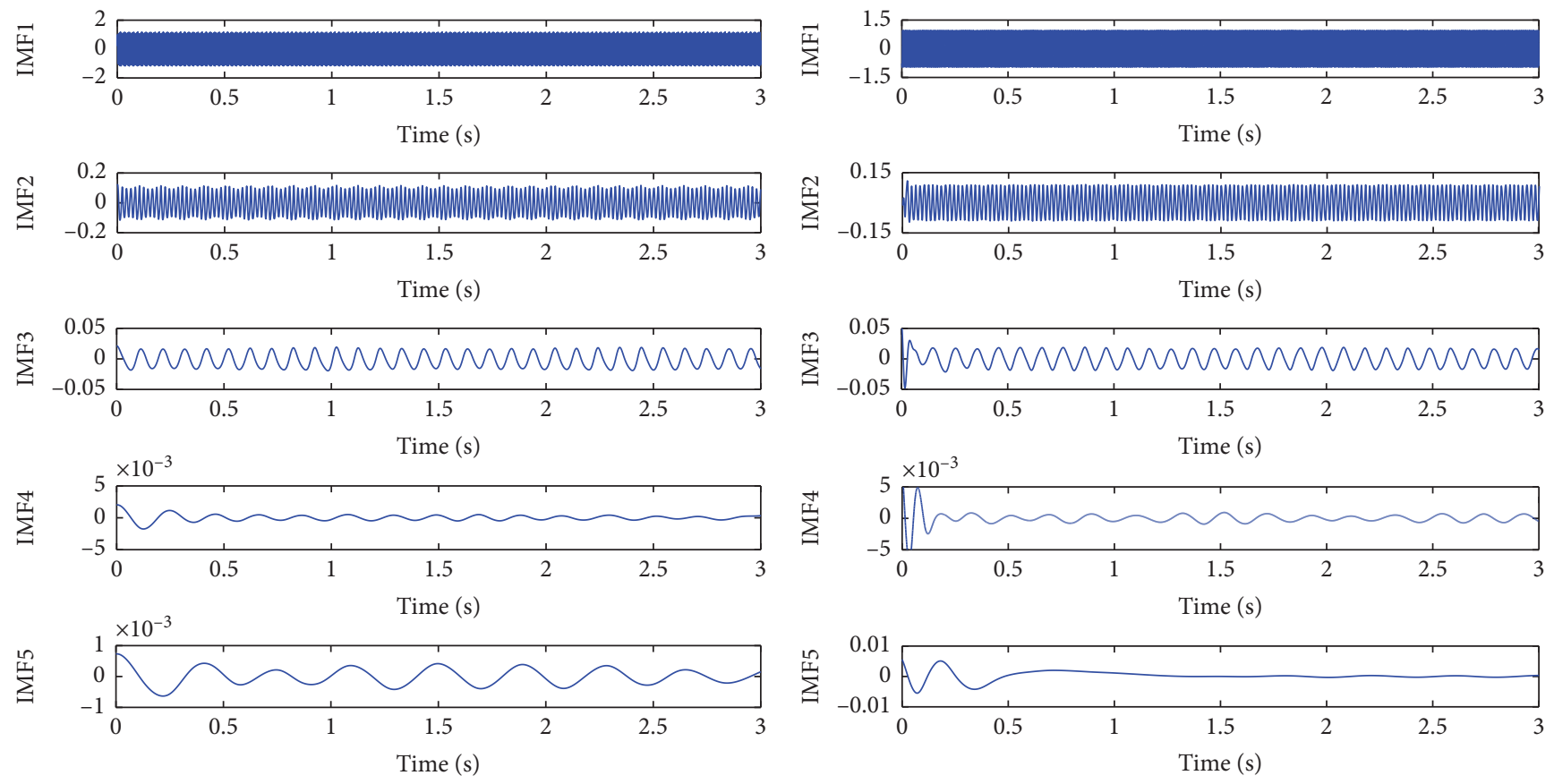

(a)

(b)
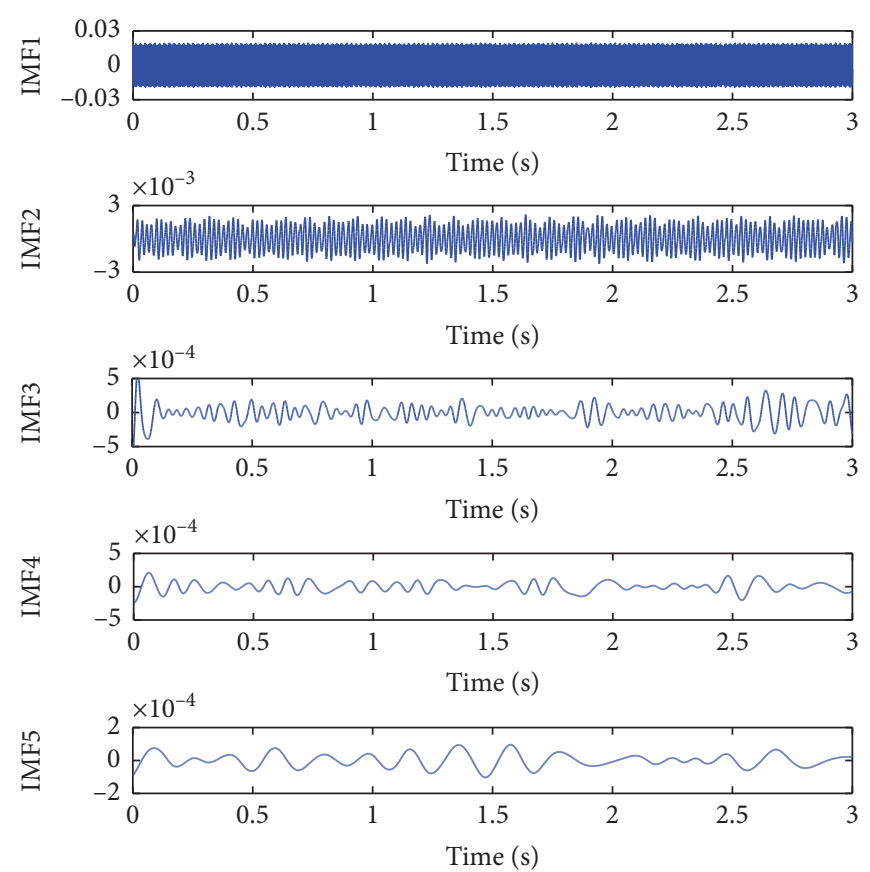

(c)

FIGURE 4: EMD results of three types of piezoelectric patch signals: (a) normal piezoelectric patch; (b) broken piezoelectric patch; (c) degummed piezoelectric patch.

the SVM under optimal parameters is obtained. The optimal fitness, average fitness, and test set identification results of each generation of intraracial particles in the three experiments are shown in Figures 6-8.

As can be seen from Figures 6-8, when the evolutionary algebra is 1 , that is, when the initial PSO did not optimize the SVM, the recognition rate of the three types of piezoelectric chips is $68 \%, 58 \%$, and $87 \%$, and the recognition rate is relatively low. After PSO optimization, only the SVD value is taken as the characteristic value in the experiment; the optimal fitness, or accuracy under cross-validation, is only $77.78 \%$, and the average fitness is about $70 \%$. The maximum value of the marginal spectrum is taken as the experiment of the eigenvalue, its optimal fitness reaches $100 \%$ at generation 50 , and the average fitness is about $80 \%$. When the SVD value and the maximum point of the marginal spectrum are 
TABLE 1: SVD values of the IMF components of the three types of piezoelectric patches.

\begin{tabular}{|c|c|c|c|c|c|}
\hline \multicolumn{6}{|c|}{ SVD values of the first 5 IMF components } \\
\hline Category & V1 & V2 & V3 & $\mathrm{V} 4$ & V5 \\
\hline \multirow{5}{*}{ Normal } & 79.98 & 5.404 & 0.2844 & 0.2511 & 0.1096 \\
\hline & 85.03 & 6.279 & 0.9019 & 0.0915 & 0.0505 \\
\hline & 88.43 & 6.885 & 1.303 & 0.0836 & 0.0723 \\
\hline & 88.92 & 7.165 & 1.452 & 0.2690 & 0.1587 \\
\hline & 85.69 & 7.163 & 1.238 & 0.1158 & 0.0588 \\
\hline \multirow{5}{*}{ Broken } & 71.86 & 3.943 & 3.246 & 1.088 & 0.6276 \\
\hline & 67.29 & 3.136 & 1.601 & 0.2429 & 0.1181 \\
\hline & 71.77 & 3.272 & 2.689 & 0.3877 & 0.3001 \\
\hline & 71.56 & 3.489 & 2.918 & 0.2870 & 0.2517 \\
\hline & 74.15 & 3.268 & 2.914 & 0.5706 & 0.4317 \\
\hline \multirow{5}{*}{ Degummed } & 1.278 & 0.1099 & 0.0337 & 0.01631 & 0.009516 \\
\hline & 1.233 & 0.1017 & 0.0231 & 0.01357 & 0.009732 \\
\hline & 1.232 & 0.0974 & 0.0145 & 0.01144 & 0.006103 \\
\hline & 1.269 & 0.1003 & 0.0484 & 0.02160 & 0.01327 \\
\hline & 1.282 & 0.0983 & 0.0126 & 0.008394 & 0.005366 \\
\hline
\end{tabular}

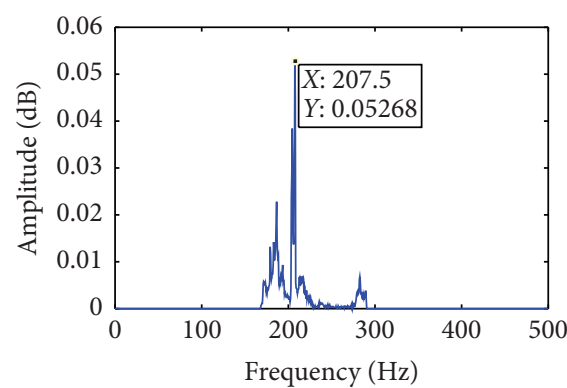

(a)

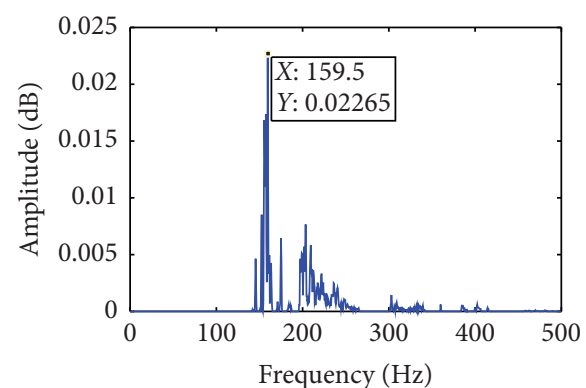

(b)

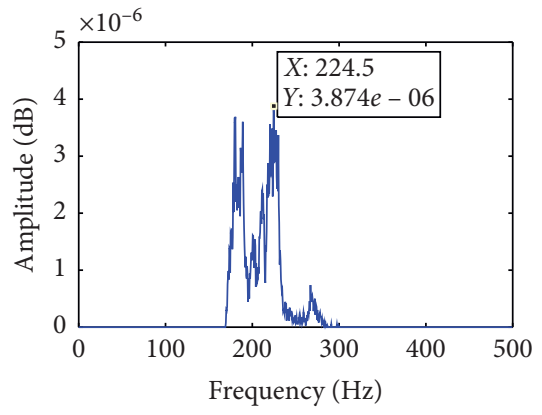

(c)

FIgURe 5: Hilbert marginal spectrum of three types of piezoelectric patch signals: (a) normal piezoelectric patch; (b) broken piezoelectric patch; (c) degummed piezoelectric patch.

TABLE 2: Hilbert marginal spectral characteristics of three types of piezoelectric patch signals.

\begin{tabular}{|c|c|c|c|c|c|c|c|c|c|c|}
\hline \multicolumn{11}{|c|}{ Hilbert marginal spectral features of the first five IMF components } \\
\hline \multirow[t]{2}{*}{ Category } & \multicolumn{5}{|c|}{$\begin{array}{l}\text { The frequency at which the maximum } \\
\text { marginal spectral amplitude is reached }\end{array}$} & \multicolumn{5}{|c|}{ The maximum value of the marginal spectrum } \\
\hline & 207.5 & 45.5 & 23.0 & 10.0 & 5.0 & 0.05268 & $3.687 E-04$ & $8.883 E-07$ & $6.005 E-06$ & $1.145 E-06$ \\
\hline \multirow{4}{*}{ Normal } & 205.0 & 55.0 & 9.0 & 4.5 & 2.5 & 0.08211 & $4.748 E-04$ & $4.220 E-05$ & $4.539 E-07$ & $3.240 E-07$ \\
\hline & 207.0 & 54.0 & 10.0 & 4.0 & 2.0 & 0.05106 & $8.134 E-04$ & $8.043 E-05$ & $6.326 E-07$ & $7.255 E-07$ \\
\hline & 208.5 & 48.5 & 10.0 & 8.0 & 1.0 & 0.04373 & $7.306 E-04$ & $1.283 E-04$ & $2.282 E-06$ & $4.673 E-06$ \\
\hline & 211.0 & 49.0 & 9.5 & 5.0 & 2.5 & 0.03623 & $8.028 E-04$ & $1.045 E-04$ & $8.916 E-07$ & $6.016 E-07$ \\
\hline \multirow{5}{*}{ Broken } & 159.5 & 131.5 & 34.5 & 52.0 & 7.0 & 0.02265 & $1.769 E-03$ & $7.381 E-04$ & $8.236 E-04$ & $1.310 E-03$ \\
\hline & 155.5 & 104.5 & 52.0 & 27.0 & 10.0 & 0.05231 & $1.568 E-05$ & $1.398 E-04$ & $2.027 E-07$ & $1.580 E-06$ \\
\hline & 155.0 & 40.0 & 49.0 & 9.5 & 10.0 & 0.04550 & $1.035 E-04$ & $1.086 E-04$ & $1.814 E-06$ & $8.508 E-06$ \\
\hline & 199.0 & 40.0 & 50.5 & 19.0 & 9.5 & 0.04392 & $1.446 E-04$ & $1.490 E-04$ & $1.259 E-06$ & $1.882 E-06$ \\
\hline & 159.0 & 46.0 & 50.0 & 18.5 & 10.0 & 0.04837 & $8.928 E-05$ & $2.126 E-04$ & $2.106 E-06$ & $1.225 E-05$ \\
\hline \multirow{5}{*}{ Degummed } & 224.5 & 41.5 & 27.5 & 19.5 & 9.0 & $3.874 \mathrm{E}-06$ & $6.291 E-08$ & $5.298 E-09$ & $2.180 E-09$ & $2.338 E-09$ \\
\hline & 179.5 & 48.5 & 27.5 & 19.0 & 10.0 & 4.547E-06 & $8.163 E-08$ & $8.107 E-09$ & $4.393 E-09$ & $2.192 E-09$ \\
\hline & 223.5 & 49.0 & 21.0 & 10.5 & 2.5 & $5.661 \mathrm{E}-06$ & $7.140 E-08$ & $2.590 E-09$ & $2.525 E-09$ & $1.116 E-09$ \\
\hline & 223.5 & 40.0 & 18.5 & 10.5 & 9.0 & $4.722 \mathrm{E}-06$ & $6.222 E-08$ & $2.888 E-08$ & $1.659 E-08$ & $8.504 E-09$ \\
\hline & 225.5 & 44.5 & 20.5 & 9.5 & 5.0 & $3.911 \mathrm{E}-06$ & $6.287 E-08$ & $1.890 E-09$ & $2.391 E-09$ & $8.536 E-10$ \\
\hline
\end{tabular}




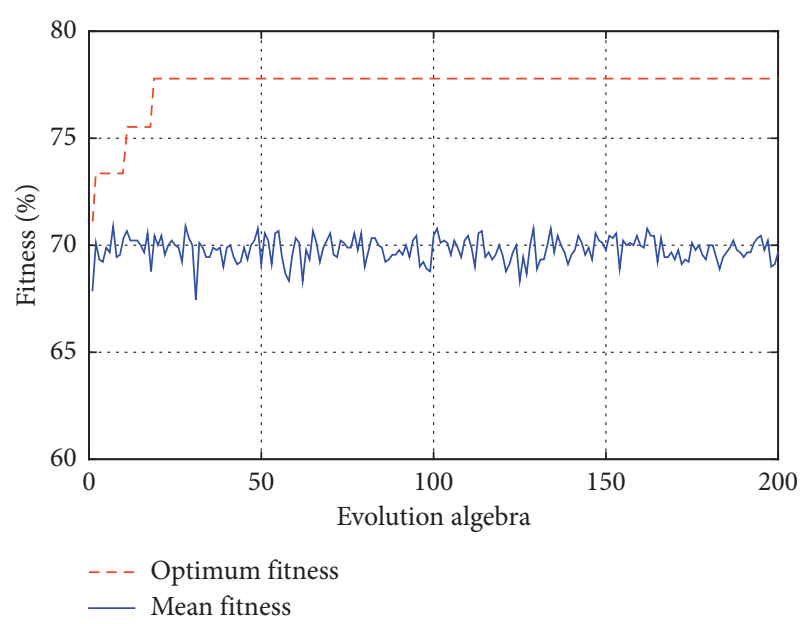

FIGURE 6: Experimental results of SVD values as eigenvalues.

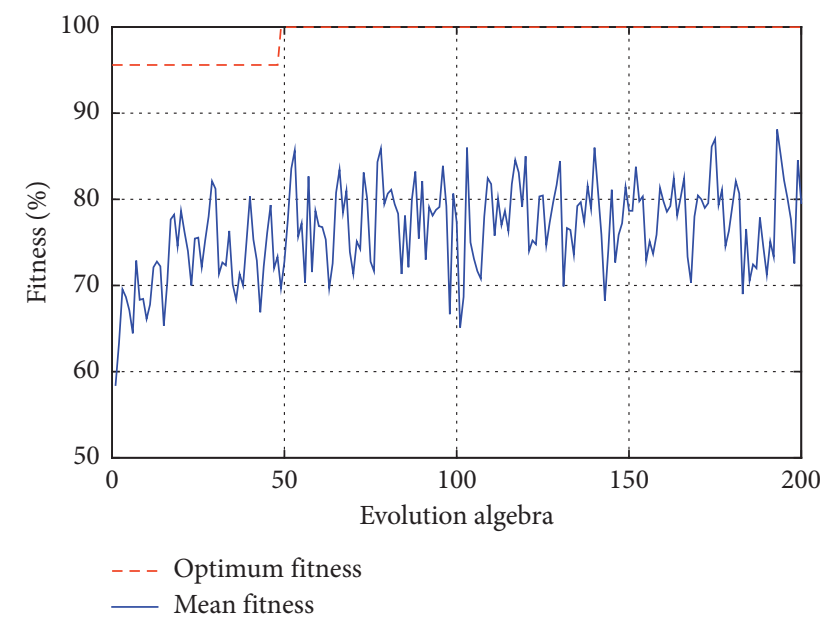

FIGURE 7: Experimental results of the Hilbert marginal spectrum as eigenvalues.

taken as the experiments of eigenvalues, the optimal fitness is $100 \%$, and the average fitness can reach about $95 \%$.

Under the aforementioned experimental conditions, multiple groups of experiments are carried out with different broken degrees and different degumming areas. The results of fracture and degumming identification are the same as those in the previous section, and they are all the best under multi-eigenvalue fusion. The experimental results also show that the degree of fracture affects the SVD value. As the degree of fracture increases, the SVD value gradually decreases within a certain range. Besides, the degree of degumming affects the marginal spectrum characteristic value. With the increase of the degumming area, the intensity of the maximum value point of the marginal spectrum decreases gradually. According to the aforementioned results, combined with the recognition result whose eigenvalue is the SVD value or Hilbert marginal spectrum feature, it can be known that, because of the large difference between the SVD value of the normal and ruptured piezoelectric patch and the SVD value of the dematerialized piezoelectric patch, the pattern recognition with the SVD

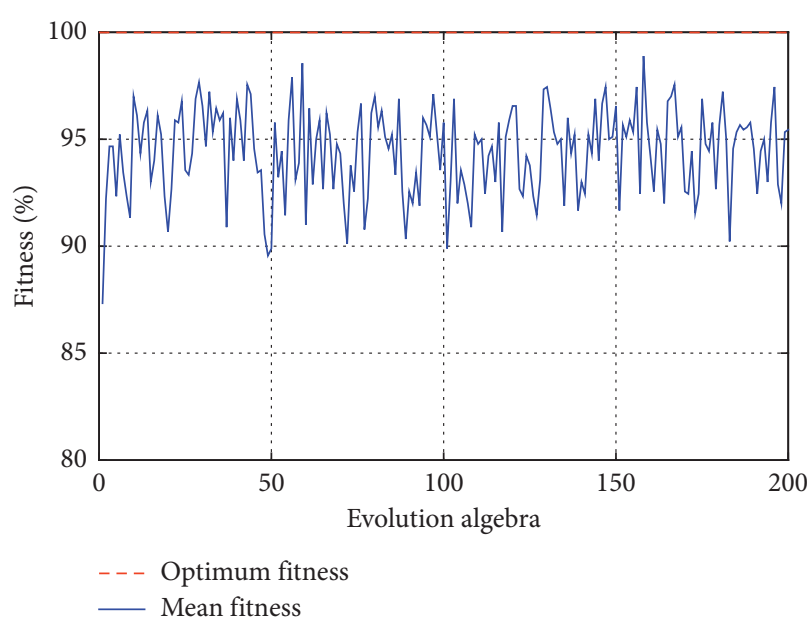

FIgURE 8: Experimental results of the SVD value and Hilbert marginal spectrum as eigenvalues.

value as the feature has too high error rate for the normal piezoelectric patch and rupture piezoelectric patch. Hilbert marginal spectrum features of the three cases are of similar orders of magnitude, but the resolution between different data is low, so the recognition accuracy is not satisfactory. By integrating the SVD value and Hilbert spectrum feature as the feature value, it has the advantages of the feature within the same order of magnitude and large data resolution. Its accuracy of classification is greatly improved, and the recognition results of the test set also verify the accuracy and superiority of the scheme.

\section{Fault Identification under Noise Signal Interference}

The recognition signal is the excitation response signal of a single square wave signal, which is different from the actual working condition. To verify the general feasibility of the fault diagnosis model and the superiority of fusion features, the study proposes the use of the square wave signal under the interference of noise signal to stimulate the stiffened plate, to simulate the actual working condition, and to carry out feature extraction and fault identification for the response signals of different fault types of piezoelectric patches, so as to verify the recognition effect.

Gaussian white noise is selected for noise signal. In order to make the experiment comparable, the experiment scheme mentioned earlier is still adopted and noise interference is added on it. That is, at first, set the duty ratio of $2.5 \%$ to $50 \%$ (every other $2.5 \%$ is a group) and the frequency as $210 \mathrm{~Hz}$, and total of 20 groups of square wave signals mixed with noise signals excite the stiffened plate respectively. A total of 60 groups of response signals are obtained from the three types of piezoelectric patches. Then, the SVD value and marginal spectrum features are extracted from the signals, and finally, different features are used for fault identification. Meanwhile, in order to improve the persuasiveness of the experiment and explore the influence of noise on the effect of fault diagnosis, Gaussian noise with the intensity of 

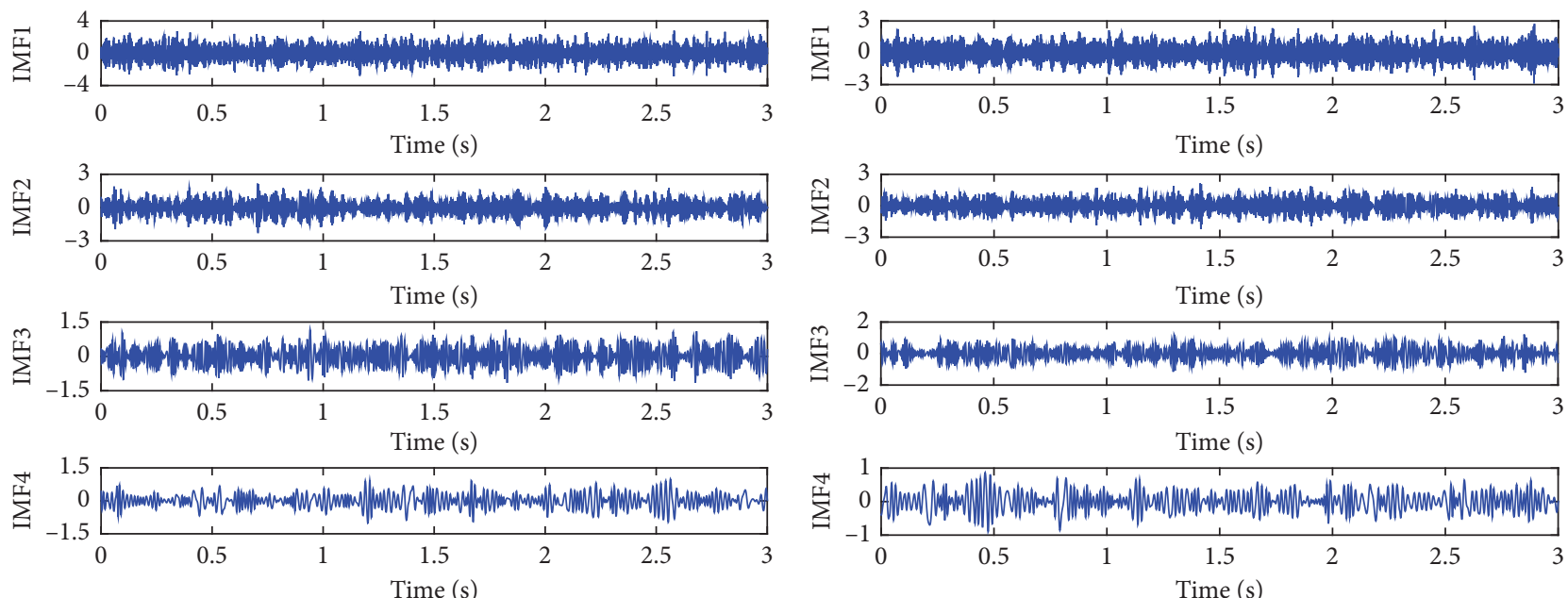

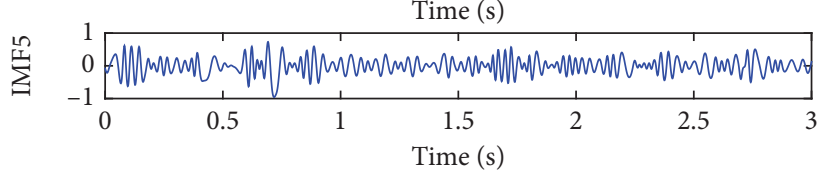

(a)

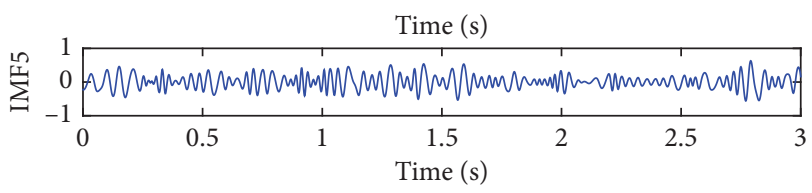

(b)
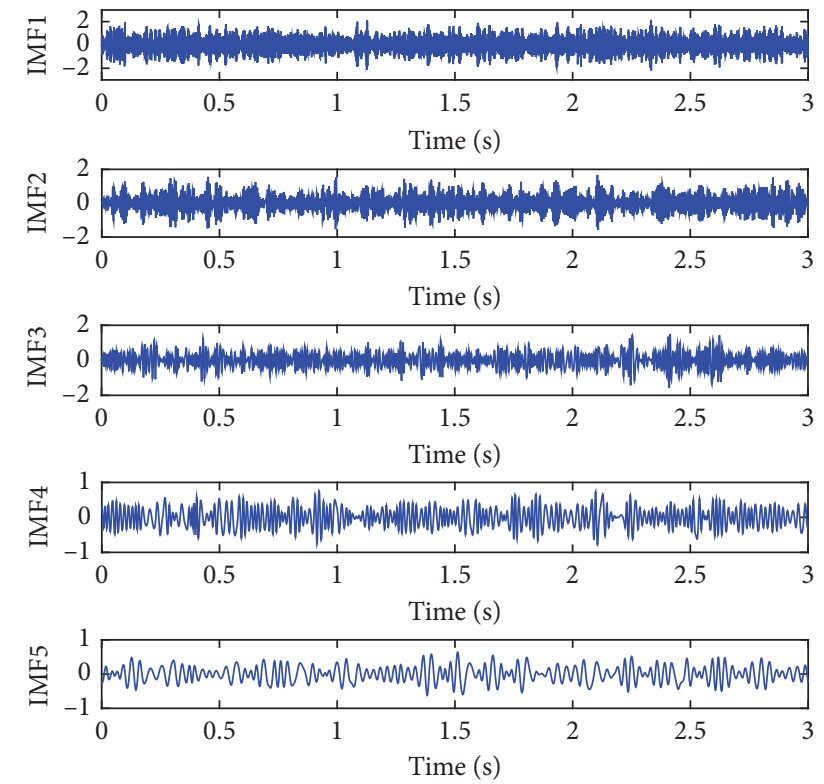

(c)

FiguRE 9: EMD results of response signals of three types of piezoelectric patches when noise signals are added: (a) normal piezoelectric patch; (b) broken piezoelectric patch; (c) degummed piezoelectric patch.

$0.5 \mathrm{dBW}-1 \mathrm{dBW}(0.05 \mathrm{dBW}$ interval) is adopted for $6 \mathrm{ex}-$ periments, respectively.

Signal feature extraction includes EMD-SVD and Hilbert marginal spectrum. EMD decomposition is carried out for the response signals under the excitation of three types of piezoelectric slice noises. The first five IMF components are obtained, as shown in Figure 9 (considering the length of the paper, IMF components of three piezoelectric patch signals are listed only under the excitation of $2.5 \%$ of the $1 \mathrm{dBW}$ noise signal).

SVD is calculated for IMF components, and SVD eigenvectors are obtained as shown in Table 3 (considering the length of the paper, SVD results of three piezoelectric patches response signals under $2.5 \%, 5 \%, 7.5 \%, 10 \%$, and $12.5 \%$ of the space-occupied example wave signals and mixed with $1 \mathrm{dBW}$ noise signals are listed).

Then, Hilbert marginal spectrum is made for IMF of each component, as shown in Figure 10 (Hilbert marginal spectrum of IMF1 component of three response signals excited by $2.5 \%$ space-occupied example wave signal mixed with $1 \mathrm{dBW}$ noise is listed only). The maximum amplitude value point of the marginal spectrum is obtained, and the results are shown in Table 4 (similarly, the results under $2.5 \%, 5 \%, 7.5 \%, 10 \%$, and $12.5 \%$ duty cycles are listed). The 
TABLE 3: SVD values of IMF components of three kinds of piezoelectric chip signals under noise signals.

\begin{tabular}{|c|c|c|c|c|c|}
\hline \multicolumn{6}{|c|}{ SVD values of the first five IMF components } \\
\hline Category & V1 & $\mathrm{V} 2$ & V3 & V4 & V5 \\
\hline \multirow{5}{*}{ Normal } & 102.96 & 68.49 & 38.59 & 29.65 & 21.02 \\
\hline & 110.99 & 65.91 & 39.81 & 30.50 & 22.85 \\
\hline & 109.15 & 68.94 & 42.12 & 31.32 & 21.36 \\
\hline & 112.88 & 67.84 & 38.71 & 30.52 & 21.85 \\
\hline & 113.75 & 70.22 & 40.20 & 26.75 & 21.58 \\
\hline \multirow{5}{*}{ Broken } & 96.70 & 60.76 & 41.65 & 29.48 & 21.24 \\
\hline & 100.16 & 63.44 & 43.02 & 29.34 & 19.88 \\
\hline & 103.56 & 61.43 & 41.58 & 31.31 & 21.54 \\
\hline & 104.46 & 62.89 & 40.55 & 30.37 & 21.72 \\
\hline & 102.14 & 67.64 & 43.06 & 28.01 & 20.42 \\
\hline \multirow{5}{*}{ Degummed } & 87.02 & 52.29 & 38.21 & 28.36 & 19.11 \\
\hline & 85.03 & 52.51 & 39.85 & 28.61 & 21.90 \\
\hline & 85.21 & 52.98 & 38.92 & 27.09 & 22.34 \\
\hline & 87.10 & 50.75 & 39.35 & 28.70 & 19.04 \\
\hline & 84.73 & 53.09 & 39.62 & 27.57 & 21.12 \\
\hline
\end{tabular}
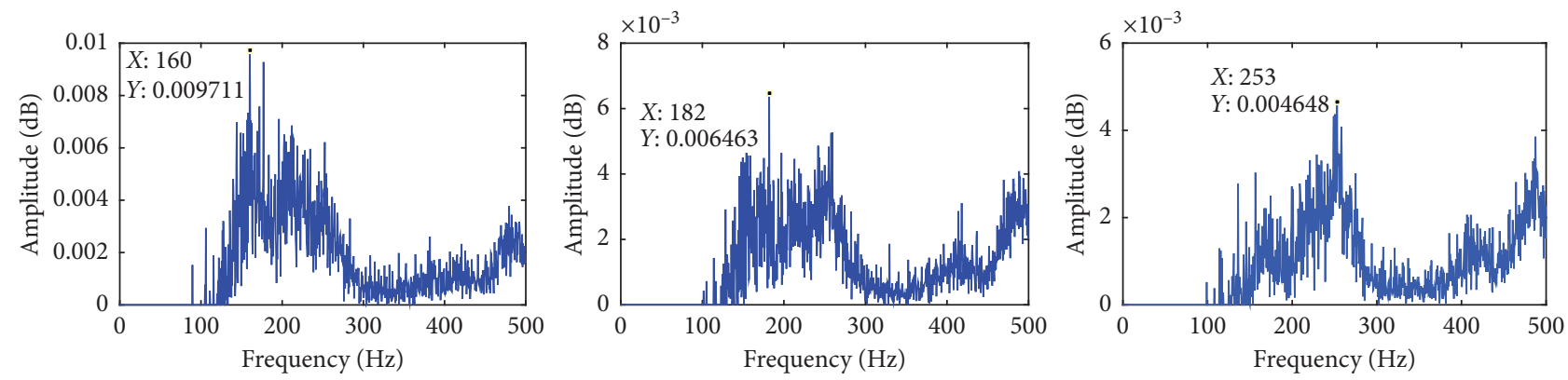

Figure 10: Hilbert marginal spectrum of three types of piezoelectric patch signals under noise signal.

TABLE 4: Hilbert marginal spectral characteristics of three types of piezoelectric patch signals under noise signal.

Hilbert marginal spectrum characteristics of the first five IMF components

Category

The frequency of the point with the maximum marginal spectral amplitude

Maximum value of marginal spectrum

\begin{tabular}{lcccccccccc}
\hline \multirow{5}{*}{ Normal } & 218 & 182.5 & 54 & 45.5 & 31.5 & 0.00844 & 0.00577 & 0.00362 & 0.00415 & 0.00303 \\
& 156.5 & 174 & 69 & 40.5 & 23.5 & 0.01252 & 0.00594 & 0.00333 & 0.00390 & 0.00353 \\
& 176.5 & 176.5 & 102 & 49.5 & 28.5 & 0.00881 & 0.00826 & 0.00473 & 0.00334 & 0.00466 \\
& 163.5 & 175.5 & 82.5 & 40.5 & 27 & 0.01284 & 0.00616 & 0.00326 & 0.00413 & 0.00321 \\
& 159 & 113.5 & 68.5 & 44.5 & 23.5 & 0.01341 & 0.00656 & 0.00409 & 0.00337 & 0.00410 \\
\hline \multirow{5}{*}{ Broken } & 174 & 169.5 & 62.5 & 41 & 15 & 0.00753 & 0.00562 & 0.00422 & 0.00350 & 0.00272 \\
& 225.5 & 217.5 & 69.5 & 47.5 & 29 & 0.00738 & 0.00537 & 0.00453 & 0.00388 & 0.00308 \\
& 165.5 & 168 & 94 & 43 & 22 & 0.00837 & 0.00461 & 0.00357 & 0.00359 & 0.00386 \\
& 152.5 & 217 & 101 & 50 & 25 & 0.00658 & 0.00550 & 0.00375 & 0.00365 & 0.00360 \\
& 163.5 & 215 & 83 & 39 & 22.5 & 0.00916 & 0.00690 & 0.00532 & 0.00449 & 0.00377 \\
\hline \multirow{5}{*}{ Degummed } & 216.5 & 129.5 & 92 & 26.5 & 27.5 & 0.00626 & 0.00455 & 0.00332 & 0.00328 & 0.00289 \\
& 258 & 173.5 & 60.5 & 45.5 & 8.5 & 0.00480 & 0.00326 & 0.00463 & 0.00345 & 0.00322 \\
& 257.5 & 169.5 & 76 & 51 & 29 & 0.00489 & 0.00373 & 0.00411 & 0.00235 & 0.00344 \\
& 255.5 & 132 & 93.5 & 29 & 17 & 0.00561 & 0.00407 & 0.00343 & 0.00297 & 0.00269 \\
& 229.5 & 161 & 85 & 57.5 & 10.5 & 0.00506 & 0.00334 & 0.00408 & 0.00324 & 0.00319 \\
\hline
\end{tabular}

results and SVD values are taken as the eigenvectors of each piezoelectric patch signal.

Similarly, the PSO-SVM algorithm described earlier is selected to identify signal features, and the parameters such as iteration times are used as described previously. The SVD value of 5 dimensions, the maximum point of the marginal spectrum of 10 dimensions, and the maximum point of the marginal spectrum of 15 dimensions are selected as the 
eigenvalues. The particle swarm optimization SVM is used to identify 60 groups of data, and the fitness function is the recognition accuracy of cross-validation of all data. After 200 iterations, the optimal and average recognition accuracy of the SVM under optimal parameters are obtained. The optimal fitness, average fitness, and test set identification results of the particles in each generation of the three experiments are shown in Figures 11-13.

Finally, the average recognition accuracy under $0.5^{\circ} \mathrm{dBW}-1^{\circ} \mathrm{dBW}$ noise is plotted, as shown in Figure 14.

It can be seen from Figures 11-13, when the evolutionary algebra is 1, that is, when the initial PSO does not optimize the SVM, the recognition rate of the three types of piezoelectric patches is $43 \%, 57 \%$, and $66 \%$, respectively, and the recognition rate is low. After PSO optimization, even under the interference of $1 \mathrm{dBW}$ noise, the average recognition accuracy of fusion features can reach $80.48 \%$. At this time, the separate SVD value features and marginal spectrum features are only $73.69 \%$ and $70.49 \%$. As can be seen from Figure 14, with the increase of mixed noise intensity, the recognition accuracy decreases gradually, which indicates that the recognition rate is affected by the interference noise intensity. Among them, the identification rate under the eigenvalues of SVD and Hilbert marginal spectrum is significantly affected. With the increase of the interference noise intensity, the recognition rate decreases greatly. Compared with the fault recognition rate under the single eigenvalue, this effect is small to the recognition rate under the fused eigenvalue. Even if the intensity is $1 \mathrm{dBW}$, the average recognition accuracy of fusion features is still about $80 \%$, and the recognition rate decreases very little. From another point of view, it shows that the advantage of the fault identification rate of fusion features is not affected by interference noise signal. In conclusion, the recognition accuracy of fused features is always higher than that of the SVD value and marginal spectrum as features.

In addition, in order to make comparative analysis, the wavelet packet transform is used to extract the characteristics of response signals of different piezoelectric patches based on the aforementioned experiments. Then, the support vector machine is also used to identify and classify the eigenvalues.

The parameters of wavelet packet adopted in this paper are set as: $\mathrm{db} 2$ wavelet, shannon entropy, and three-layer decomposition. The specific steps are as follows [4]:

(1) The response signals of each piezoelectric sensor are decomposed into three layers, and the characteristics of eight frequencies under the three layers are extracted.

(2) Reconstruct the wavelet packet decomposition coefficient, and extract the signal $S_{3 i}(i=0, \ldots, 7)$ of each frequency band. The reconstruction coefficient diagram of the wavelet packet is shown in Figure 15 (only the reconstruction coefficients of response signals stimulated by square wave signals with a duty cycle of $2.5 \%$ and a strength of $1 \mathrm{dBW}$ noise interference signal are listed).

(3) Calculate the total signal energy of each frequency band, and set the energy corresponding to $S_{3 i}$ as $E_{3 i}$,

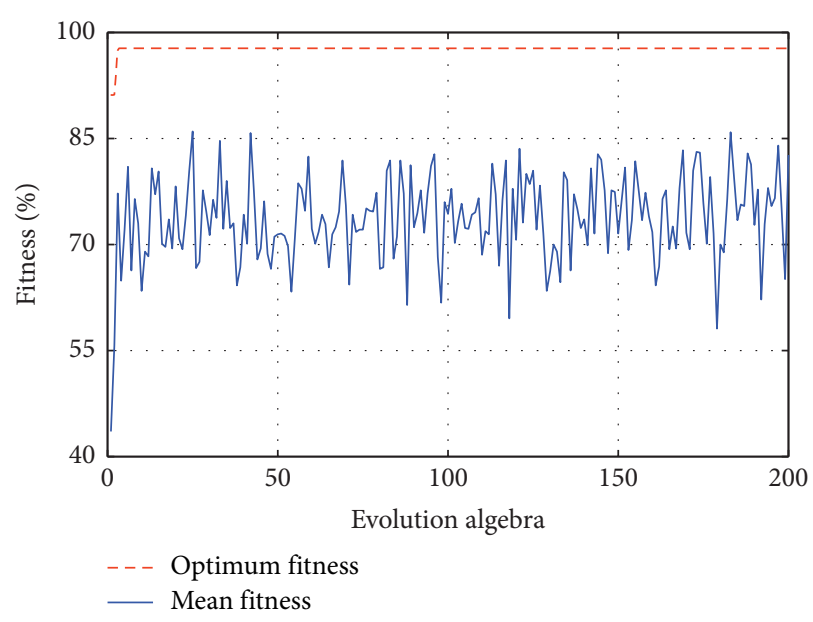

FIGURE 11: Identification results of the SVD value as the eigenvalue when noise signal is added into excitation.

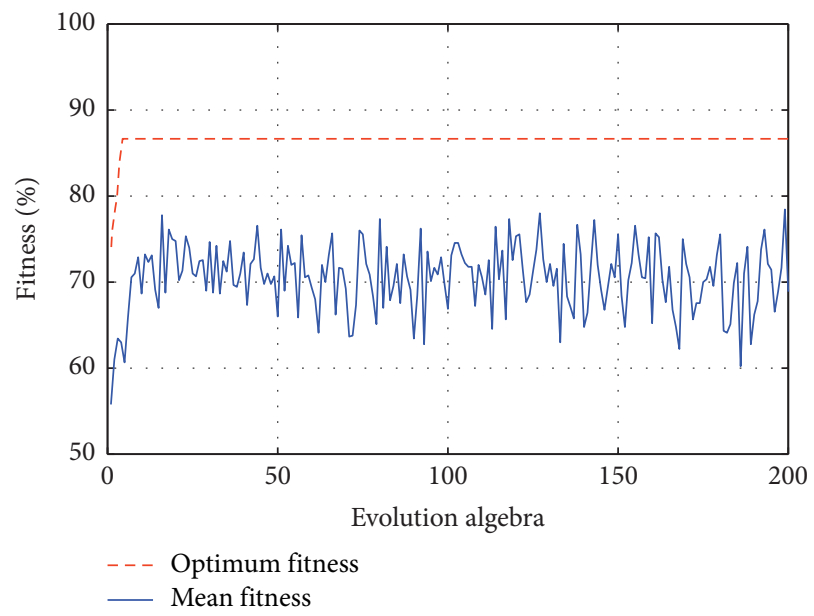

FIgURE 12: Identification results of Hilbert marginal spectrum as the eigenvalue when noise signal is added into excitation.

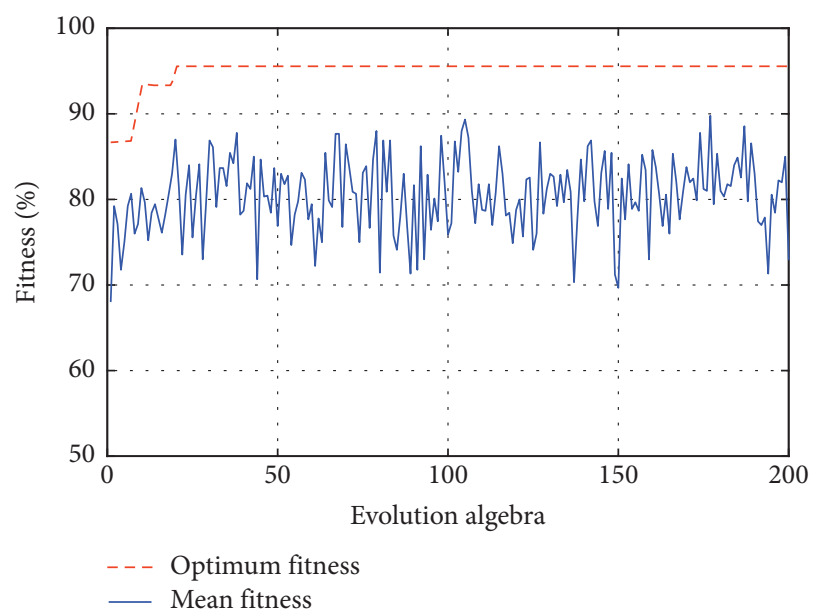

FIGURE 13: Identification results of the SVD value and Hilbert marginal spectrum as the eigenvalue when noise signal is added into excitation. 


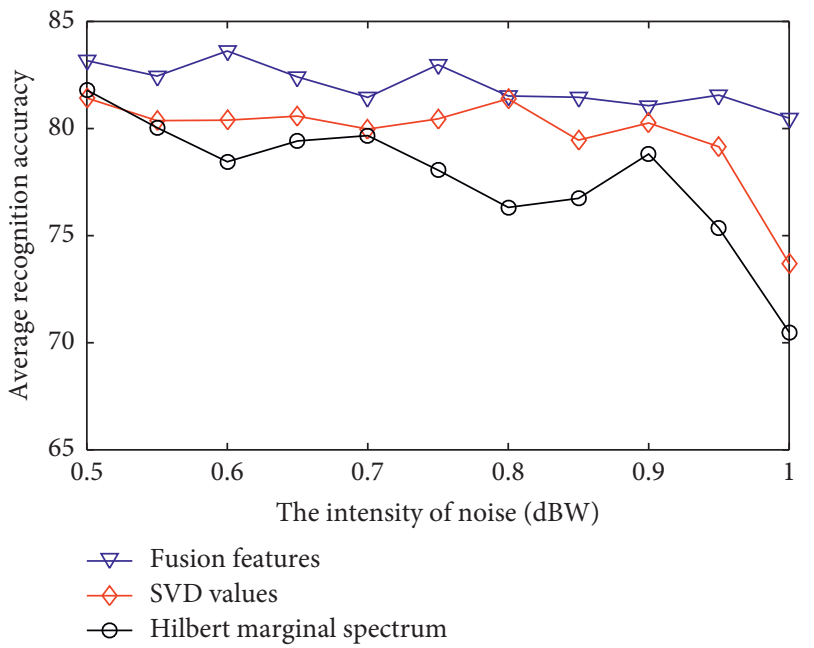

FIgURE 14: Recognition effect of different eigenvalues when noise signal of different intensities is added into excitation.
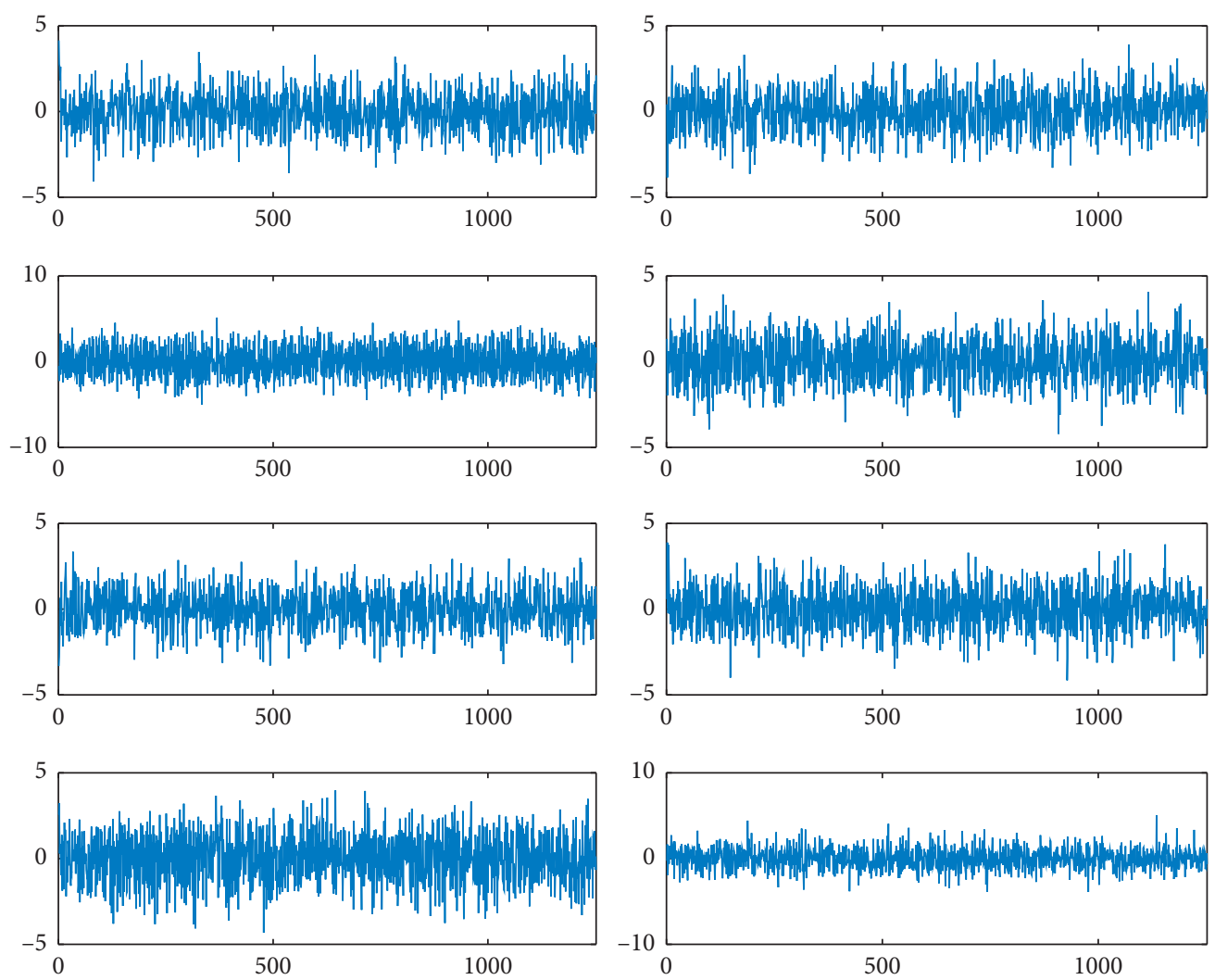

(a)

Figure 15: Continued. 

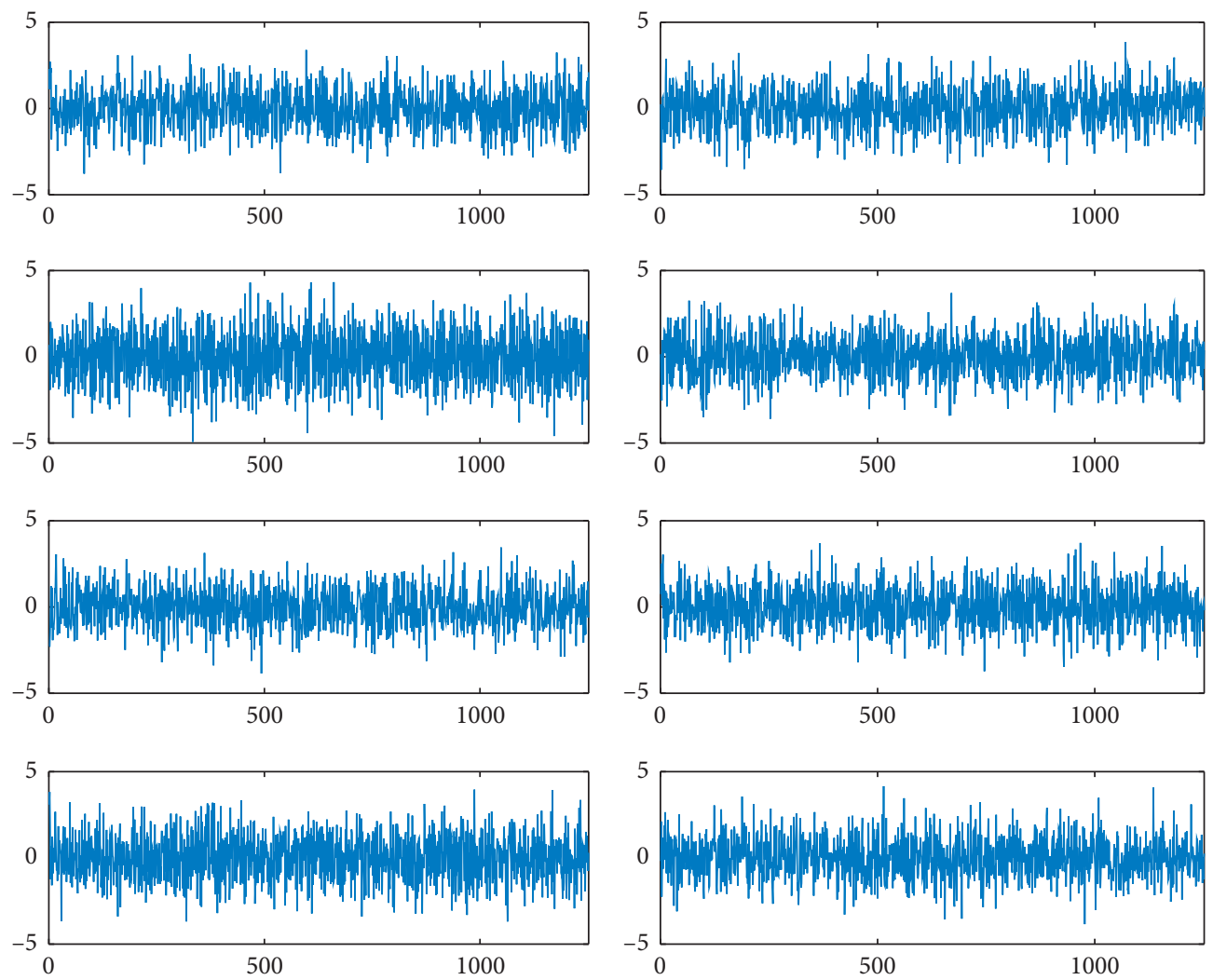

(b)
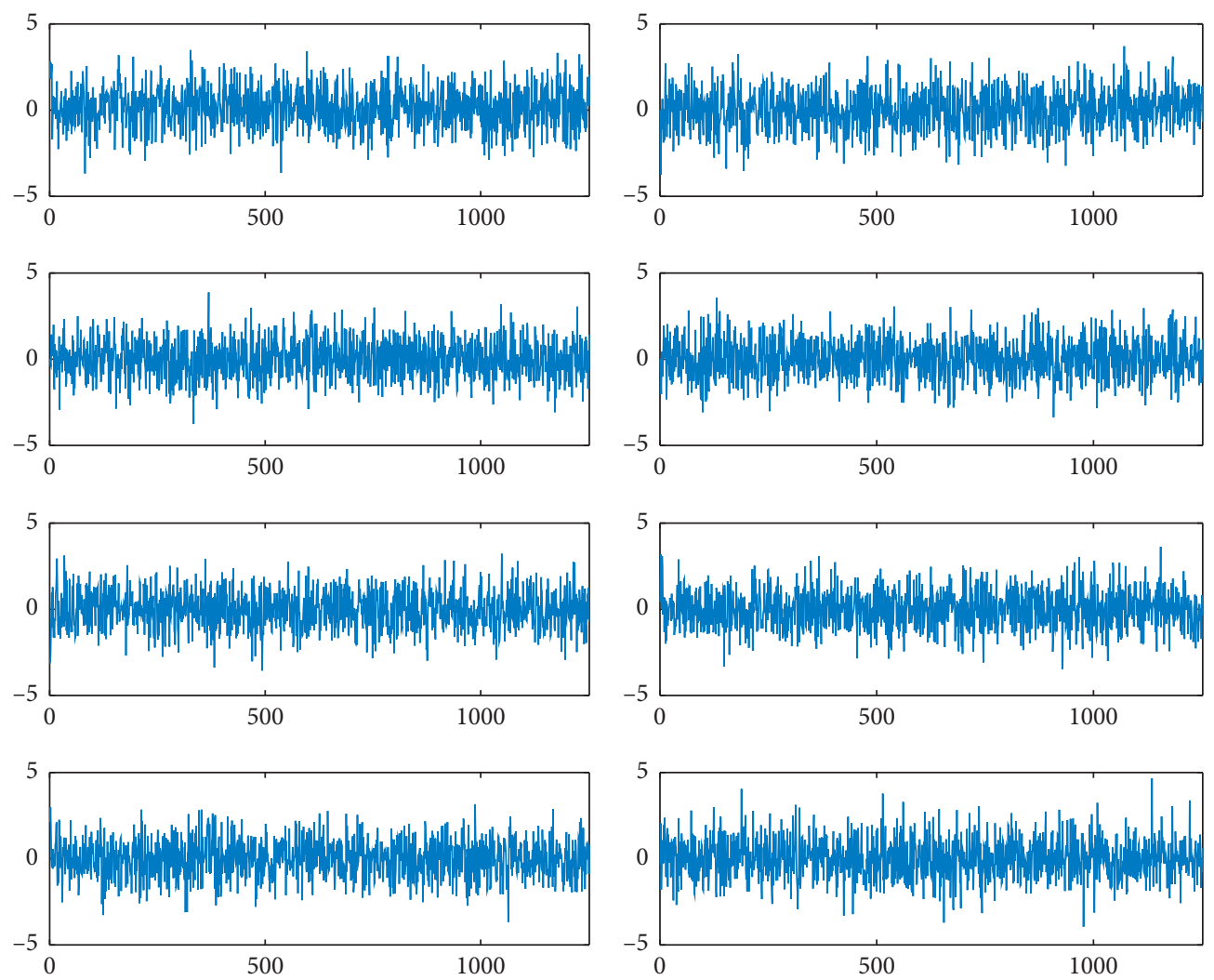

(c)

FIGURE 15: Response signal reconstruction coefficients of piezoelectric patches: (a) normal piezoelectric patch; (b) broken piezoelectric patch; (c) degummed piezoelectric patch. 
TABLE 5: Signal energy characteristics of three kinds of piezoelectric patches.

\begin{tabular}{|c|c|c|c|c|c|c|c|c|}
\hline \multicolumn{9}{|c|}{ Normalized eigenvectors } \\
\hline \multirow{5}{*}{ Normal } & 0.3461 & 0.3528 & 0.3508 & 0.3457 & 0.3607 & 0.3640 & 0.3523 & 0.3557 \\
\hline & 0.3457 & 0.3523 & 0.3531 & 0.3452 & 0.3603 & 0.3630 & 0.3535 & 0.3550 \\
\hline & 0.3458 & 0.3524 & 0.3533 & 0.3462 & 0.3604 & 0.3623 & 0.3527 & 0.3550 \\
\hline & 0.3461 & 0.3530 & 0.3494 & 0.3455 & 0.3608 & 0.3633 & 0.3531 & 0.3567 \\
\hline & 0.3459 & 0.3526 & 0.3516 & 0.3458 & 0.3605 & 0.3639 & 0.3523 & 0.3555 \\
\hline \multirow{5}{*}{ Broken } & 0.3465 & 0.3535 & 0.3482 & 0.3457 & 0.3613 & 0.3636 & 0.3528 & 0.3566 \\
\hline & 0.3460 & 0.3529 & 0.3519 & 0.3455 & 0.3607 & 0.3627 & 0.3530 & 0.3553 \\
\hline & 0.3461 & 0.3530 & 0.3491 & 0.3459 & 0.3609 & 0.3643 & 0.3522 & 0.3565 \\
\hline & 0.3463 & 0.3534 & 0.3486 & 0.3457 & 0.3611 & 0.3631 & 0.3531 & 0.3566 \\
\hline & 0.3463 & 0.3531 & 0.3491 & 0.3456 & 0.3610 & 0.3631 & 0.3534 & 0.3564 \\
\hline \multirow{5}{*}{ Degummed } & 0.3468 & 0.3534 & 0.3488 & 0.3457 & 0.3612 & 0.3637 & 0.3523 & 0.3561 \\
\hline & 0.3468 & 0.3534 & 0.3488 & 0.3457 & 0.3612 & 0.3637 & 0.3523 & 0.3561 \\
\hline & 0.3467 & 0.3534 & 0.3488 & 0.3457 & 0.3612 & 0.3637 & 0.3524 & 0.3561 \\
\hline & 0.3467 & 0.3534 & 0.3488 & 0.3457 & 0.3612 & 0.3637 & 0.3523 & 0.3561 \\
\hline & 0.3467 & 0.3534 & 0.3488 & 0.3457 & 0.3612 & 0.3637 & 0.3524 & 0.3561 \\
\hline
\end{tabular}

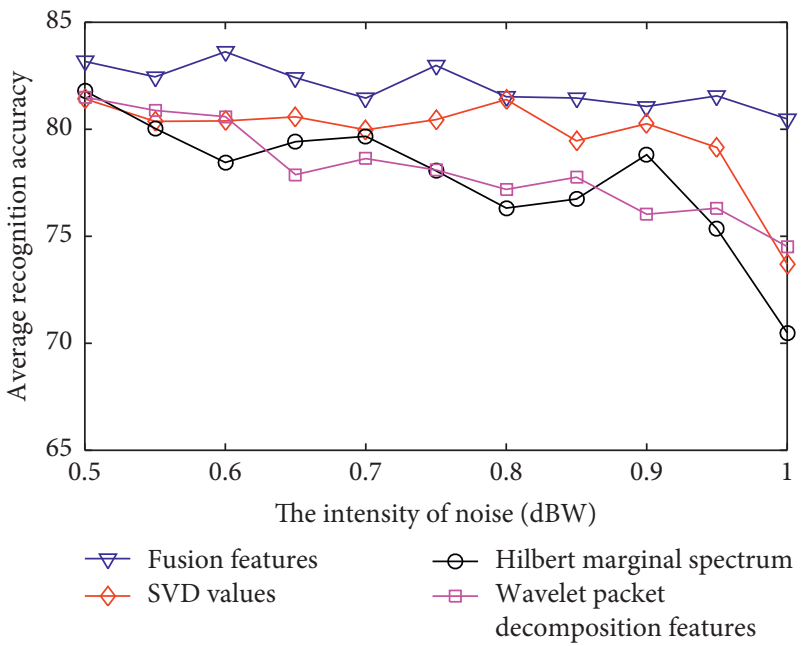

FIgURE 16: Recognition effect of different eigenvalues when noise signal of different intensities is added into excitation.

which $x_{i k}(i=0, \ldots, 7 ; k=1, \ldots, n)$ is the amplitude of $E_{3 i}$ discrete points.

(4) Different frequency band energies are used to construct eigenvectors and carry out normalization processing, and the results are shown in Table 5 (only the normalized eigenvector results of three kinds of piezoelectric patches signals under square wave excitation with duty cycles of $2.5 \%, 5 \%, 7.5 \%, 10 \%$, and $12.5 \%$ mixed with $1 \mathrm{dBW}$ noise are listed).

Similarly, the particle swarm optimization SVM algorithm described earlier is selected to identify the signal features obtained by wavelet packet transformation, and the parameters such as the number of iterations are followed as mentioned earlier. In the noise of $0.5-1 \mathrm{dBW}$ (interval $0.05 \mathrm{dBW}$ ), the recognition results of wavelet packet transform features are compared with the recognition results of different features to draw a line diagram, as shown in Figure 16.

As can be seen from Figure 16, the recognition result of eigenvalues obtained by the wavelet packet decomposition method is not very ideal. Moreover, the recognition accuracy decreases greatly with the increase in noise intensity, and the recognition accuracy is greatly affected by environmental interference. By contrast, the accuracy of fusion eigenvalue recognition is higher, moreover, the accuracy of recognition is less disturbed by environmental noise, and the recognition result is relatively stable.

\section{Conclusions}

In this study, the piezoelectric sensor in vibration control of the stiffened plate is taken as the research object. Two kinds of piezoelectric sensor faults, fracture and degumming, are simulated. EMD-SVD and Hilbert marginal spectrum are used to extract the characteristics of voltage signals under different excitation. The PSO-optimized SVM algorithm is used to identify fault types of different eigenvalues. Experimental results show that the recognition effect of multifeature fusion is the best, no matter under non-noise interference or noise interference. In the case of non-noise, the average accuracy can reach $95 \%$. In the case of noise interference of $0.5 \mathrm{dBW}-1 \mathrm{dBW}$, fusion features are superior 
to SVD value features and marginal spectrum features as features alone. The experimental results show that the fault diagnosis method of piezoelectric sensor patches based on multifeature fusion and PSO-SVM is effective and has certain advantages. At the same time, this method lays a good foundation for the future research on active faulttolerant control of stiffened plate structure vibration.

\section{Data Availability}

The data used to support the findings of this study are included within the supplementary information files.

\section{Conflicts of Interest}

The authors declare that there are no conflicts of interest regarding the publication of this article.

\section{Acknowledgments}

This work was funded by the project of National Natural Science Foundation of China (51305003), Anhui Postdoctoral Fund Project (2017B172), and Anhui University of Science and Technology National Nature Fund Pre-Research Project (2016yz004).

\section{Supplementary Materials}

1-Supplementary Materials is the response signal characteristics of three types of piezoelectric sensors in 20 experiments, where the response signal characteristics were the SVD value and Hilbert marginal spectrum. In the 20 experiments, the stiffened plate was excited by square wave signals with a duty cycle of $2.5-50 \%$ (every $2.5 \%$ is a group) and a frequency of $210 \mathrm{~Hz}$. The 2-Supplementary Materials is based on the experiment in the 1Supplementary Materials to add the noise interference, that is, first set up between $2.5 \%$ and $50 \%$ duty cycle (every $2.5 \%$ is a group), frequency of $210 \mathrm{~Hz}$ group, a total of 20 square wave signal with noise, respectively, to incentive of stiffened plate, three types of piezoelectric patches, get 60 group response signals, and then the signal for SVD value and marginal spectrum feature extraction, and the feature extraction results were the submitted supplementary materials. (Supplementary Materials)

\section{References}

[1] Y. Li, M. Xu, H. Zhao et al., "Research on bearing diagnosis method based on hierarchical fuzzy entropy and improved support vector machine," Journal of Vibration Engineering, vol. 29, no. 1, pp. 184-192, 2016.

[2] Y. Fu, L. Jia, Y. Qin et al., "Fault diagnosis method for rolling bearing based on LMD-CM-PCA," Journal of Vibration, Testing and Diagnosis, vol. 37, no. 2, pp. 249-255, 2017.

[3] H. Yang and H.-X. Pan, "The adaptive analysis of shock signals on the basis of improved morlet wavelet clusters," Shock and Vibration, vol. 2018, Article ID 9892713, 13 pages, 2018.

[4] T. Ma, F. Du, N. Xiong et al., "Fault diagnosis of piezoelectric sensors in vibration control of flexible manipulators," Journal of Electronic Measurement and Instrument, vol. 28, no. 12, pp. 1408-1413, 2014.

[5] X. Zhang, Y. Liang, J. Zhou, and Y. Zang, "A novel bearing fault diagnosis model integrated permutation entropy, ensemble empirical mode decomposition and optimized SVM," Measurement, vol. 69, pp. 164-179, 2015.

[6] C. Lu, Z. Y. Wang, W. L. Qin et al., "Fault diagnosis of rotary machinery components using a stacked denoising autoencoder-based health state identification," Signal Processing, vol. 130, pp. 377-388, 2016.

[7] Z. Gao and D. W. C. Ho, "State/noise estimator for descriptor systems with application to sensor fault diagnosis," IEEE Transactions on Signal Processing, vol. 54, no. 4, pp. 13161326, 2006.

[8] L. Yang, C. Shen, X. Jin et al., "Intelligent diagnosis of piezoelectric acceleration sensor faults," Instrumentation Technology and Sensors, vol. 30, no. 10, pp. 115-118, 2016.

[9] L. Yang, W. Chen, and H. Wang, "Fault diagnosis and isolation of vehicle hydraulic active suspension based on residual information," China Mechanical Engineering, vol. 23, no. 14, pp. 1746-1752, 2012.

[10] J. Sun, J. Qi, L. Fei et al., "Self-damage detection of piezoelectric components based on impedance technology," Journal of Vibration, Testing and Diagnosis, vol. 31, no. 4, pp. 454-458, 2011.

[11] F. Hou, J. Chen, and G. Dong, "Weak fault feature extraction of rolling bearings based on globally optimized sparse coding and approximate SVD," Mechanical Systems and Signal Processing, vol. 111, 2018.

[12] C. Huang, H. Song, W. Lei, Z. Niu, Y. Meng, and D. Younesian, "Instantaneous amplitude-frequency feature extraction for rotor fault based on BEMD and Hilbert transform," Shock and Vibration, vol. 2019, Article ID 1639139, 19 pages, 2019.

[13] Z. Chen, S. Deng, X. Chen, C. Li, R.-V. Sanchez, and H. Qin, "Deep neural networks-based rolling bearing fault diagnosis," Microelectronics Reliability, vol. 75, pp. 327-333, 2017.

[14] M. Kang, J. Kim, and J.-M. Kim, "Reliable fault diagnosis for incipient low-speed bearings using fault feature analysis based on a binary bat algorithm," Information Sciences, vol. 294, pp. 423-438, 2015.

[15] S. Lu, J. Wang, and Y. Xue, "Study on multi-fractal fault diagnosis based on EMD fusion in hydraulic engineering," Applied Thermal Engineering, vol. 103, pp. 798-806, 2016.

[16] C. Yi, J. Lin, W. Zhang, and J. Ding, "Faults diagnostics of railway axle bearings based on IMF's confidence index algorithm for ensemble EMD," Sensors, vol. 15, no. 5, pp. 10991-11011, 2015.

[17] J. Cheng, D. Yu, J. Tang et al., "Application of SVM and SVD technique based on EMD to the fault diagnosis of the rotating machinery," Shock \& Vibration, vol. 16, no. 1, pp. 89-98, 2013.

[18] Z. Li, R. Outbib, S. Giurgea et al., "Online implementation of SVM based fault diagnosis strategy for PEMFC systems," Applied Energy, vol. 164, no. 2, pp. 284-293, 2016.

[19] M. Li, "The application of PCA and SVM in rolling bearing fault diagnosis," Advanced Materials Research, vol. 430-432, pp. 1163-1166, 2012.

[20] J. Zheng and J. Cheng, "Improved hilbert-huang transform and its applications to rolling bearing fault diagnosis," Journal of Mechanical Engineering, vol. 51, no. 1, pp. 138-145, 2015.

[21] Y. Huang, D. Wu, Z. Zhang, H. Chen, and S. Chen, "EMDbased pulsed TIG welding process porosity defect detection and defect diagnosis using GA-SVM," Journal of Materials Processing Technology, vol. 239, pp. 92-102, 2017. 
[22] Z. Liye, Y. Wei, and Y. Ruqiang, "Gearbox fault diagnosis using complementary ensemble empirical mode decomposition and permutation entropy," Shock and Vibration, vol. 2016, Article ID 3891429, 8 pages, 2016.

[23] T. Zhe, L. Wei, Z. Bo et al., "Bearing fault diagnosis based on domain adaptation using transferable features under different working conditions," Shock and Vibration, vol. 2018, Article ID 6714520, 12 pages, 2018.

[24] Y. Zhang, P. Zhang, H. Wang et al., "Feature extraction method for rolling bearing vibration signals based on VMD and Volterra prediction model," Journal of Vibration and Shock, vol. 37, no. 3, pp. 129-135, 2018.

[25] X. Zhang, L. Tang, P. Wang et al., "Fault diagnosis of acoustic emission of rolling bearing based on SVD and fast kurtogram algorithm," Journal of Vibration and Shock, vol. 33, no. 10, pp. 101-105, 2014.

[26] H. Jiang, J. Chen, G. Dong, T. Liu, and G. Chen, "Study on Hankel matrix-based SVD and its application in rolling element bearing fault diagnosis," Mechanical Systems and Signal Processing, vol. 52-53, no. 1, pp. 338-359, 2015.

[27] B. Yang, R. Liu, and X. Chen, "Fault diagnosis for a wind turbine generator bearing via sparse representation and shiftinvariant K-SVD," IEEE Transactions on Industrial Informatics, vol. 13, no. 3, pp. 1321-1331, 2017.

[28] X. G. Xi, "SEMG movement pattern recognition of hand based on correlation analysis and SVM," Journal of Electronics \& Information Technology, vol. 30, no. 10, pp. 2315-2319, 2008.

[29] H. Wu, L. Jianxin, L. Lai et al., "Fault pattern recognition based on EMD-SVD model and SVM rolling bearing," Noise and Vibration Control, vol. 31, no. 2, pp. 89-93, 2011.

[30] D. He, J. Peng, J. Hu et al., "Fault diagnosis of CS-SVM bearing based on improved FOA optimization," Journal of Vibration and Shock, vol. 18, no. 15, pp. 108-114, 2018. 


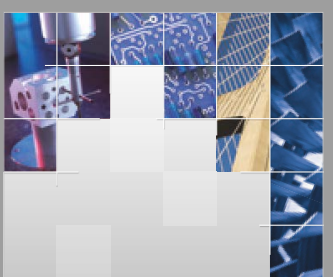

\section{Enfincering}
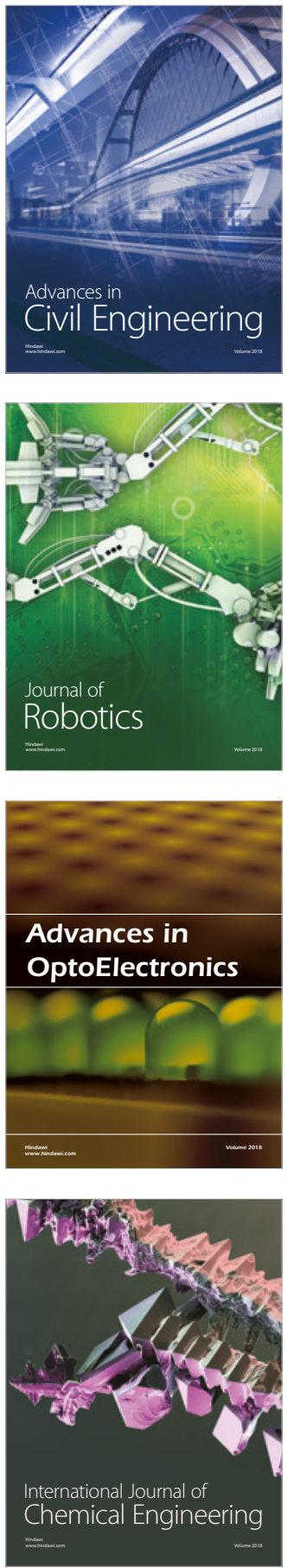

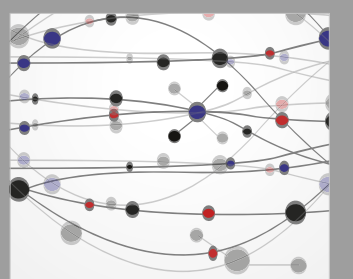

\section{Rotating \\ Machinery}

The Scientific World Journal

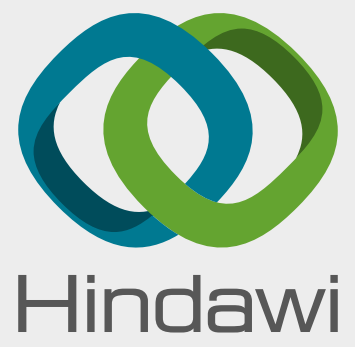

Submit your manuscripts at

www.hindawi.com
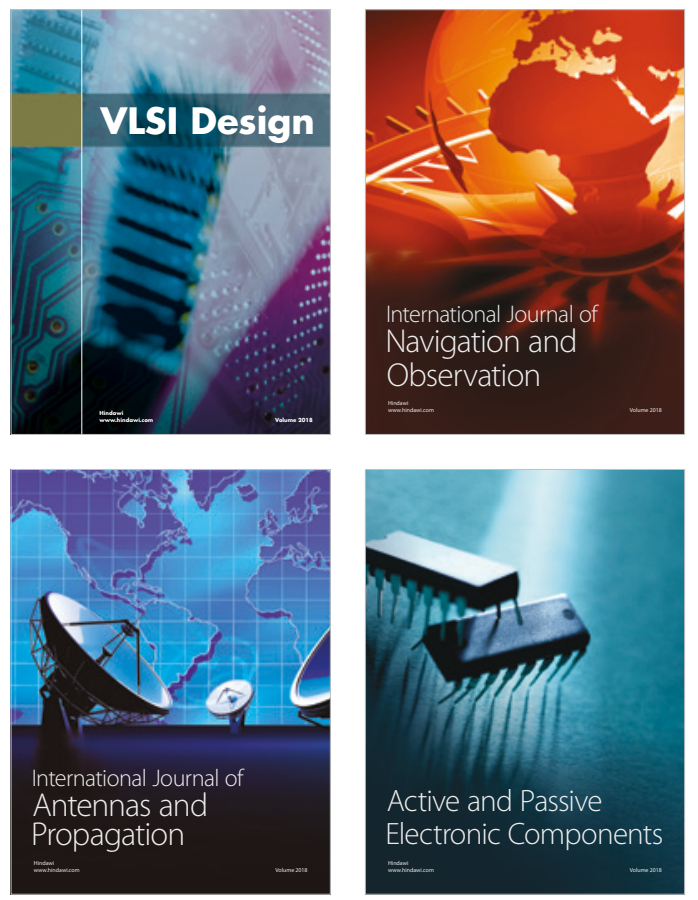
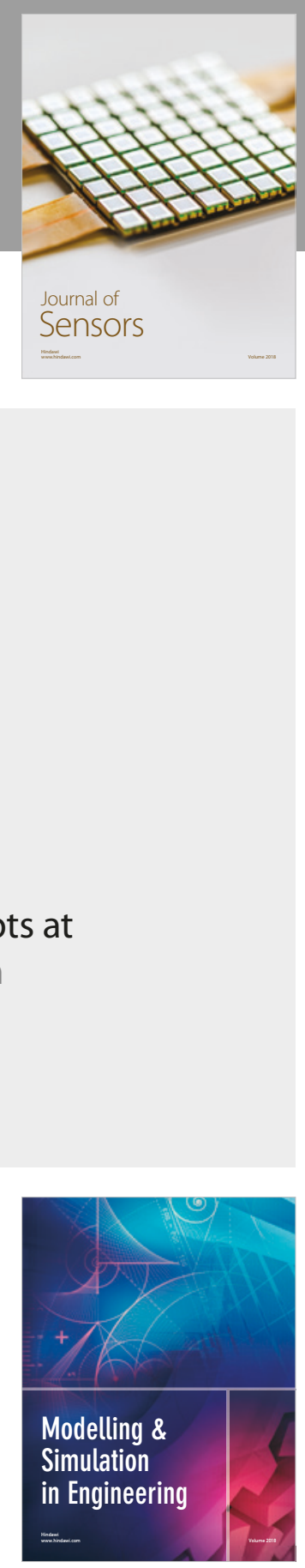

\section{Advances \\ Multimedia}
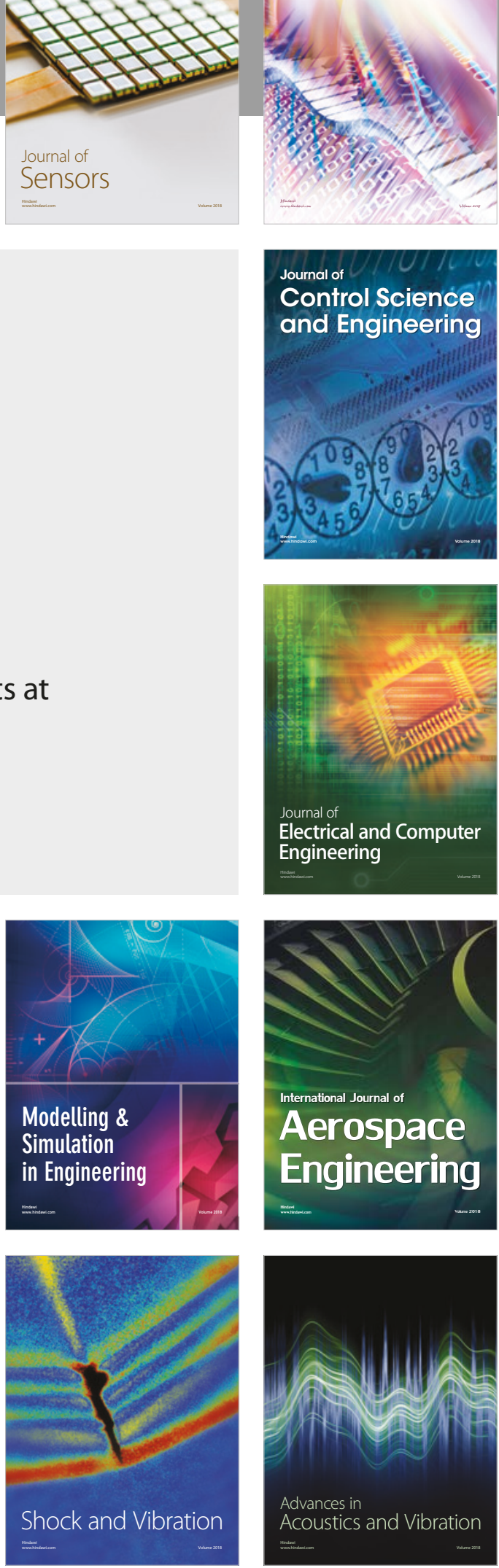